\title{
Free and Forced Vibration Analysis of Airtight Cylindrical Vessels with Doubly Curved Shells of Revolution by Using Jacobi-Ritz Method
}

\author{
Fuzhen Pang, ${ }^{1}$ Haichao Li, ${ }^{1}$ Kwangnam Choe, ${ }^{2}$ Dongyan Shi, ${ }^{3}$ and Kwanghun Kim ${ }^{2}$ \\ ${ }^{1}$ College of Shipbuilding Engineering, Harbin Engineering University, Harbin 150001, China \\ ${ }^{2}$ Department of Light Industry Machinery Engineering, Pyongyang University of Mechanical Engineering, \\ Pyongyang 999093, Democratic People's Republic of Korea \\ ${ }^{3}$ College of Mechanical and Electrical Engineering, Harbin Engineering University, Harbin 150001, China
}

Correspondence should be addressed to Haichao Li; lihaichao@hrbeu.edu.cn

Received 19 May 2017; Accepted 19 September 2017; Published 9 November 2017

Academic Editor: Lorenzo Dozio

Copyright $@ 2017$ Fuzhen Pang et al. This is an open access article distributed under the Creative Commons Attribution License, which permits unrestricted use, distribution, and reproduction in any medium, provided the original work is properly cited.

\begin{abstract}
This paper presents free and forced vibration analysis of airtight cylindrical vessels consisting of elliptical, paraboloidal, and cylindrical shells by using Jacobi-Ritz Method. In this research, the theoretical model for vibration analysis is formulated by Flügge's thin shell theory and the solution is obtained by Rayleigh-Ritz method. The vessel structure is divided into shell components (i.e., ellipsoid, parabolic, and cylinder) and their segments, and each displacement field of shell segments is represented by the Jacobi polynomials and the standard Fourier series. The continuous conditions at the interface are modeled by using the spring stiffness technique. The reliability and the accuracy of the present method are verified by comparing the results of the proposed method with the results of the previous literature and the finite element method (FEM). Moreover, some numerical results for free and forced vibration of elliptical-cylindrical-elliptical vessel (ECE vessel) and paraboloidal-cylindrical-elliptical vessel (PCE vessel) are reported.
\end{abstract}

\section{Introduction}

The coupled shell consisting of a cylindrical shell and doubly curved shells of revolution are widely used in aerospace and marine structures and civil and mechanical engineering. For example, hermetic capsules, pressure vessels, and storage vessels can be modeled as elliptical-cylindrical-elliptical shell structures; rockets and submarines can be viewed as parabolic-cylindrical-elliptical shell structures. So it is important to analyze the vibration characteristics of the coupled shell mentioned above.

To emphasize the characteristic and purpose of this paper, the existing research related to this paper is reviewed and shown below: Leissa [1] and Qatu [2] analyzed the classical thin shell theories (Love's, Reissner's, Naghdi's, Sanders', and Flügge's, etc.) and the thick shell theories. Based on these theories, many researchers have proposed free and forced vibration analysis methods for some structures. Liew and
Lim [3] proposed a pb-2 Ritz method to study the free vibration of the doubly curved shallow shells in form of a rectangular plane. Tan [4] presented an efficient substructuring analysis method to investigate the free vibration characteristics for doubly curved shells of revolution based on the first-order shear deformation shell theory and the classical thin shell theory. Tang et al. [5] researched the free and forced vibration analysis of multistepped circular cylindrical shell with arbitrary boundary conditions with the employment of the method of reverberation-ray matrix. Xie et al. [6] presented a semianalytic method to analyze free and forced vibrations of combined conical-cylindricalspherical shells with ring stiffeners and bulkheads. Xie et al. [7] presented an analytic method for free and forced vibration analysis of stepped conical shells with general boundary conditions. Qatu and Asadi [8] used the Ritz method to present vibration frequencies for spherical, cylindrical, and hyperbolic paraboloidal shells with 21 possible boundary 
conditions by using thin shallow shell theory. Zhang et al. [9] proposed an analytical method to study the free and forced vibration behaviors of a submerged finite elliptic cylindrical shell. Jiang et al. [10] derived a unified solution for the vibration analysis of doubly curved shallow shells with arbitrary elastic supports by using an improved trigonometric series and the Rayleigh-Ritz method. Messina [11] studied the free vibrations of multilayered doubly curved shells based on a mixed variational approach and global piecewise-smooth functions. Poultangari and Nikkhah-Bahrami [12] developed the Vectorial-wave method (VWM) to study free and forced vibrations of cylindrical shells in the presence of dampers at supports. Chen et al. [13] presented an analytic method to analyze free and forced vibration characteristics of ringstiffened combined conical-cylindrical shells with arbitrary boundary conditions. Tornabene et al. [14-16] studied the dynamic behavior of functionally graded or laminated composite doubly curved shells and panels of revolution by using the Generalized Differential Quadrature (GDQ) method. Chen et al. [17] presented wave based method to analyze the free and forced vibration of cylindrical shells with discontinuity in thickness. Ma et al. [18] presented a free and forced vibration analysis of coupled conical-cylindrical shells with arbitrary boundary conditions using a modified Fourier-Ritz method. Dozio [19] deals with the formulation of advanced two-dimensional Ritz-based models for accurate prediction of natural frequencies of thin and thick sandwich plates core made of functionally graded material. In [20, 21], research results are presented on dynamic behavior of the composite laminated or the functionally graded material (FGM) doubly curved shells and panels of revolution with arbitrary boundary conditions. In literatures the displacement fields are represented by using several auxiliary functions and a standard Fourier series, the classical boundary conditions and the general elastic restraint are imitated by the coupling spring stiffness technique, and a unified numerical analysis model is presented to solve the free vibration of doubly curved shells and panels by using the Rayleigh-Ritz method. Dozio [22] used the state-space levy's method to solve the free vibration problem of specially orthotropic multilayered cylindrical and spherical panels. Dozio and Carrera [23] described a variable-kinematic Ritz formulation to accurately predict free vibration of thick and thin, rectangular, and skew multilayered plates with clamped, free, and simply supported boundary conditions.

Galletly and Mistry [24] investigated the vibration characteristics of the closed cylindrical shells with cones, hemispheres, ellipsoids, and so on by using the finite difference method. Irie et al. [25] represented the vibration equation of the shells as a combined set of first-order differential equations by using the transfer matrix method, and the research studied vibration characteristics of the coupled conical-cylindrical and annular plate-cylindrical shell. Qu et al. [26] presented a domain decomposition technique for solving vibration problems of uniform and stepped cylindrical shells with arbitrary boundary conditions. Wong and Sze [27] investigated low frequency vibration of the torsion free axisymmetric modes of thin cylindrical shell with hemispherical caps vibrating in vacuo by using membrane approximation. Rougui et al. [28] investigated the nonlinear free and forced vibrations of simply supported thin circular cylindrical shells using Lagrange's equations and an improved transverse displacement expansion. Efraim and Eisenberger [29] studied the dynamic behaviors of coupled shells made of conical, cylindrical, and plate segments by using a dynamic stiffness matrix method. Caresta and Kessissoglou [30] presented the vibration characteristics of isotropic coupled conical-cylindrical shells by using a wave solution and a power series expansion method. Lee [31, 32] presented the axisymmetric free vibration analysis of sphere, hemisphere and the joined spherical-cylindrical shell structures by using a pseudospectral method. Qu et al. [3336] proposed a kind of domain decomposition method to study the vibration characteristics of various combination shell structures consisting of conical, cylindrical, spherical shell, and ring stiffeners. Chen et al. [13] presented an analytic method for free and forced vibration analysis of ring-stiffened combined conical-cylindrical shells with arbitrary boundary conditions by using Flügge's theory and the smeared out method.

As discussed above, many of the vibration analyses focus on the simple doubly curved shells, while the study on the vibration analysis for their coupled shell structures is relatively small. Furthermore, most of the mentioned researches studied the vibration problems of the jointed shell structures consisting of cylindrical, conical, and spherical shells. And the authors prefer the free vibration analysis instead of the forced vibration analysis of the structures, especially for the structures of doubly curved shells of revolution. However, the results of these studies provide the feasibility for solving the free and forced vibration problems of airtight cylindrical vessels with doubly curved shells of revolution.

The main purpose of this work is to present a generalized and unified Jacobi-Ritz formulation to investigate the free and forced vibration characteristics of airtight cylindrical vessels consisting of the elliptical, paraboloidal, and cylindrical shells. This paper is characterized by not only simplifying the calculation by applying the Rayleigh-Ritz method and the penalty function method, but also generalizing the selection of the permissible displacement function by using the Jacobi polynomial. Flügge's thin shell theory is employed to do vibration analysis for theoretical model; the continuous conditions at the interface are modeled by using the spring stiffness technique. Based on the domain decomposition method [33-36], the vessel structures are divided into ellipsoid, parabola, cylinder, and their segments, and displacement functions are represented by the Jacobi polynomials and the standard Fourier series. The comparisons with the results of the previous paper or the finite element method (FEM) are presented, and some numerical results for free and forced vibration of the elliptical-cylindrical-elliptical shell and the paraboloidal-cylindrical-elliptical shell are reported.

\section{Theoretical Formulations}

2.1. Geometry of the Airtight Cylindrical Vessel. The geometric model for the airtight cylindrical vessel with doubly curved shell of revolution is shown in Figure 1. 


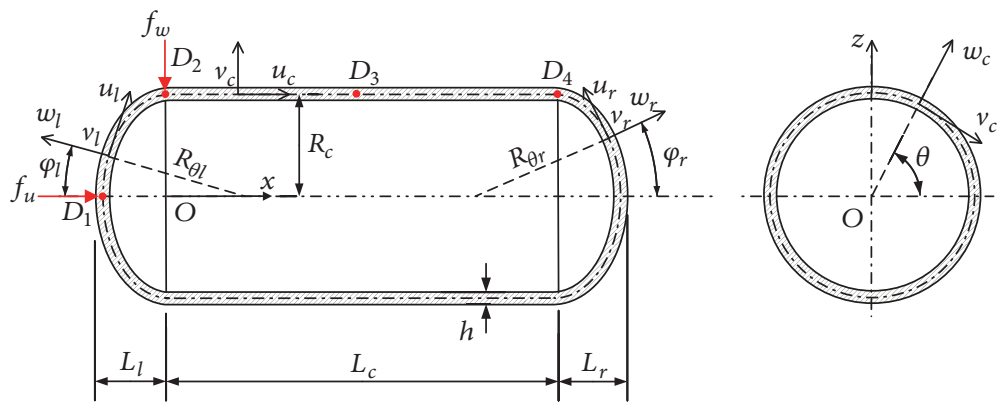

FIGURE 1: Cross section and coordinate systems of an airtight cylindrical vessel.

The airtight cylindrical vessel is divided into its components, that is, the elliptical, cylindrical, and paraboloidal shell, depending on the position of the junctions. The orthogonal coordinate systems $\left(\varphi_{l, r}, \theta_{l, r}, z_{l, r}\right)$ for the left and right doubly curved shells are fixed in the middle surface of shells, where $\varphi$ are the coordinates along the meridional direction, $\theta$ are the circumferential coordinates, and $z$ are normal coordinates. Also the cylindrical shell is described by a cylindrical coordinate system $\left(x, \theta_{c}, z_{c}\right)$, where the subscripts $l, c$, and $r$ represent the left, cylindrical, and right shell, respectively. We need to remember that there are two main curvatures radii (curvature radius of the meridional direction $R_{\varphi}$, curvature radius of the circumferential direction $R_{\theta}$ ) in geometry of the doubly curved shells $[20,21]$, and two curvatures radii of the elliptical shell and the paraboloidal shell can be expressed as follows:

Elliptical shell:

$$
\begin{aligned}
& R_{\varphi}(\varphi)=\frac{a^{2} b^{2}}{\sqrt{\left(a^{2} \sin ^{2} \varphi+b^{2} \cos ^{2} \varphi\right)^{3}}} ; \\
& R_{\theta}(\varphi)=\frac{a^{2}}{\sqrt{a^{2} \sin ^{2} \varphi+b^{2} \cos ^{2} \varphi}}
\end{aligned}
$$

where $a$ and $b$ are the lengths of the semimajor and semiminor axes of the elliptic meridian, which are given by $a=R_{c}$ and $b=L_{l, r}$, respectively.

Paraboloidal shell:

$$
\begin{aligned}
& R_{\varphi}(\varphi)=\frac{k}{2 \cos ^{3} \varphi} ; \\
& R_{\theta}(\varphi)=\frac{k}{2 \cos \varphi},
\end{aligned}
$$

where $k$ is the characteristic parameter of the parabolic meridian.

Specially,

$$
\begin{aligned}
k & =\frac{R_{c}^{2}}{L_{l}}, \\
\varphi_{p} & =\arctan \left(\frac{2 R_{c}}{k}\right),
\end{aligned}
$$

where $\varphi_{p}$ is the coordinate angle along the meridian at the junction between the paraboloidal shell and the cylindrical shell. Also, in this study, the spherical shell is considered to be a special case of the elliptical shell; the extents of the coordinate angle $\varphi$ along the meridian for elliptical shell and the paraboloidal shell are, respectively, defined by $\varphi \in$ $[0, \pi / 2]$ and $\varphi \in\left[0, \varphi_{p}\right]$. Regardless of each shell component, it is assumed that all shells are composed of homogeneous and isotropic materials; the elastic modulus $E$, mass density $\rho$, Poisson's ratio $\nu$, and thickness $h$ have the same values, respectively. The displacement of each shell component is presented by $u_{\xi}, v_{\xi}$, and $w_{\xi}(\xi=l, c, r)$ at coordinate systems. In order to increase the accuracy of calculations, these shell components may be further decomposed into $N_{l}$ left, $N_{c}$ cylindrical, and $N_{r}$ right shell component segments along the meridian or the axial of revolution (see Qu et al. [34]).

2.2. Energy Functions of the Airtight Cylindrical Vessel. In this work, Flügge's thin shell theory [1] is employed to define the relationships between strain and displacement of the middle surface of the $i$ th segment in the $\xi$ shell component. They are written as follows:

$$
\begin{aligned}
\varepsilon_{\varphi_{\xi}}^{i}= & \frac{1}{A} \frac{\partial u_{\xi}^{i}}{\partial \varphi_{\xi}}+\frac{v_{\xi}^{i}}{A B} \frac{\partial A}{\partial \theta_{\xi}}+\frac{w_{\xi}^{i}}{R_{\varphi_{\xi}}}, \\
\varepsilon_{\theta_{\xi}}^{i}= & \frac{1}{B} \frac{\partial v_{\xi}^{i}}{\partial \theta_{\xi}}+\frac{u_{\xi}^{i}}{A B} \frac{\partial B}{\partial \varphi_{\xi}}+\frac{w_{\xi}^{i}}{R_{\theta_{\xi}}}, \\
\varepsilon_{\varphi_{\theta_{\xi}}}^{i}= & \frac{A}{B} \frac{\partial}{\partial \theta_{\xi}}\left(\frac{u_{\xi}^{i}}{A}\right)+\frac{B}{A} \frac{\partial}{\partial \varphi_{\xi}}\left(\frac{v_{\xi}^{i}}{B}\right), \\
k_{\varphi_{\xi}}^{i}= & \frac{1}{A} \frac{\partial}{\partial \varphi_{\xi}}\left(\frac{u_{\xi}^{i}}{R_{\varphi_{\xi}}}-\frac{1}{A} \frac{\partial w_{\xi}^{i}}{\partial \varphi_{\xi}}\right) \\
& +\frac{1}{A B}\left(\frac{v_{\xi}^{i}}{R_{\theta_{\xi}}}-\frac{1}{B} \frac{\partial w_{\xi}^{i}}{\partial \theta_{\xi}}\right) \frac{\partial A}{\partial \theta_{\xi}}, \\
k_{\theta_{\xi}}^{i}= & \frac{1}{B} \frac{\partial}{\partial \theta_{\xi}}\left(\frac{v_{\xi}^{i}}{R_{\theta_{\xi}}}-\frac{1}{B} \frac{\partial w_{\xi}^{i}}{\partial \theta_{\xi}}\right) \\
& +\frac{1}{A B}\left(\frac{u_{\xi}^{i}}{R_{\varphi_{\xi}}}-\frac{1}{A} \frac{\partial w_{\xi}^{i}}{\partial \varphi_{\xi}}\right) \frac{\partial B}{\partial \varphi_{\xi}},
\end{aligned}
$$




$$
\begin{aligned}
k_{\varphi \theta_{\xi}}^{i}= & \frac{A}{B} \frac{\partial}{\partial \theta_{\xi}}\left[\frac{1}{A}\left(\frac{u_{\xi}^{i}}{R_{\varphi_{\xi}}}-\frac{1}{A} \frac{\partial w_{\xi}^{i}}{\partial \varphi_{\xi}}\right)\right] \\
& +\frac{B}{A} \frac{\partial}{\partial \varphi_{\xi}}\left[\frac{1}{B}\left(\frac{v_{\xi}^{i}}{R_{\theta_{\xi}}}-\frac{1}{B} \frac{\partial w_{\xi}^{i}}{\partial \theta_{\xi}}\right)\right] \\
& +\frac{1}{R_{\varphi_{\xi}}}\left(\frac{1}{B} \frac{\partial u_{\xi}^{i}}{\partial \theta_{\xi}}-\frac{v_{\xi}^{i}}{A B} \frac{\partial B}{\partial \varphi_{\xi}}\right) \\
& +\frac{1}{R_{\theta_{\xi}}}\left(\frac{1}{A} \frac{\partial v_{\xi}^{i}}{\partial \varphi_{\xi}}-\frac{u_{\xi}^{i}}{A B} \frac{\partial A}{\partial \theta_{\xi}}\right),
\end{aligned}
$$

where $\varepsilon_{\varphi_{\xi}}^{i}$, $\varepsilon_{\theta_{\xi}}^{i}$, and $\varepsilon_{\varphi \theta_{\xi}}^{i}$ are strain components of the meridional, circumferential, and shearing direction in the middle surface of the shell, $k_{\varphi_{\xi}}^{i}, k_{\theta_{\xi}}^{i}$, and $k_{\varphi \theta_{\xi}}^{i}$ are the curvature changes of the middle surface in the shell, and $u_{\xi}^{i}, v_{\xi}^{i}$, and $w_{\xi}^{i}$ are the displacement components in the meridional, circumferential, and normal directions of the shell, respectively. In above equations, the superscript $i$ represents the $i$ th segment in the $\xi$ shell component and the subscript $\xi(\xi=l, c, r)$ represents the left, cylindrical, and right shell, respectively. Also, $A$ and $B$ are the Lamé parameters; $R_{\varphi_{\xi}}$ and $R_{\theta_{\xi}}$ indicate the curvature radius in the meridional directions and circumferential directions of middle surface, respectively. The coordinate system and geometric parameters of each shell components in Figure 1 are defined as follows:

(1) Left shell:

$$
\begin{aligned}
\varphi_{\xi} & =\varphi_{l}, \\
\theta_{\xi} & =\theta_{l}, \\
R_{\varphi_{\xi}} & =R_{\varphi_{l}}, \\
R_{\theta_{\xi}} & =R_{\theta_{l}}, \\
A & =R_{\varphi_{l}}, \\
B & =R_{\theta_{l}} \sin \varphi_{l},
\end{aligned}
$$

(2) Cylindrical shell:

$$
\begin{aligned}
\varphi_{\xi} & =x, \\
\theta_{\xi} & =\theta_{c}, \\
R_{\varphi_{\xi}} & =\infty, \\
R_{\theta_{\xi}} & =R_{c}, \\
A & =1, \\
B & =R_{c},
\end{aligned}
$$

(3) Right shell:

$$
\begin{gathered}
\varphi_{\xi}=\varphi_{r}, \\
\theta_{\xi}=\theta_{r}, \\
R_{\varphi_{\xi}}=R_{\varphi_{r}},
\end{gathered}
$$

$$
\begin{aligned}
R_{\theta_{\xi}} & =R_{\theta_{r}}, \\
A & =R_{\varphi_{r}}, \\
B & =R_{\theta_{r}} \sin \varphi_{r} .
\end{aligned}
$$

With reference to the Flügge's thin shell theory, the normal and shear strains of the shell components are expressed as follows:

$$
\begin{aligned}
& e_{\varphi_{\xi}}^{i}=\frac{1}{1+z_{\xi} / R_{\varphi_{\xi}}}\left(\varepsilon_{\varphi_{\xi}}^{i}+z_{\xi} k_{\varphi_{\xi}}^{i}\right), \\
& e_{\theta_{\xi}}^{i}=\frac{1}{1+z_{\xi} / R_{\theta_{\xi}}}\left(\varepsilon_{\theta_{\xi}}^{i}+z_{\xi} k_{\theta_{\xi}}^{i}\right), \\
& \gamma_{\varphi \theta_{\xi}}^{i} \\
& \quad=\frac{1}{\left(1+z_{\xi} / R_{\varphi_{\xi}}\right)\left(1+z_{\xi} / R_{\theta_{\xi}}\right)}\left[\left(1-\frac{z_{\xi}^{2}}{R_{\varphi_{\xi}} R_{\theta_{\xi}}}\right) \varepsilon_{\varphi \theta_{\xi}}^{i}\right. \\
& \left.\quad+z_{\xi}\left(1+\frac{z_{\xi}}{2 R_{\varphi_{\xi}}}+\frac{z_{\xi}}{2 R_{\theta_{\xi}}}\right) k_{\varphi \theta_{\xi}}^{i}\right],
\end{aligned}
$$

where $-h / 2 \leq z_{\xi} \leq h / 2$.

From Hooke's law on the relationship between stress and strain, the corresponding stresses are expressed as follows:

$$
\left\{\begin{array}{c}
\sigma_{\varphi_{\xi}}^{i} \\
\sigma_{\theta_{\xi}}^{i} \\
\sigma_{\varphi \theta_{\xi}}^{i}
\end{array}\right\}=\left[\begin{array}{ccc}
Q_{11} & Q_{12} & 0 \\
Q_{21} & Q_{22} & 0 \\
0 & 0 & Q_{66}
\end{array}\right]\left\{\begin{array}{c}
e_{\varphi_{\xi}}^{i} \\
e_{\theta_{\xi}}^{i} \\
\gamma_{\varphi \theta_{\xi}}^{i}
\end{array}\right\},
$$

where $\sigma_{\varphi_{\xi}}^{i}, \sigma_{\theta_{\xi}}^{i}$, and $\sigma_{\varphi \theta_{\xi}}^{i}$ are the normal and shear stresses and $Q_{i, j}(i, j=1,2,6)$ are the constants indicating the relationship between stress and strain, which are defined as follows:

$$
\begin{aligned}
& Q_{11}=Q_{22}=\frac{E}{1-v^{2}}, \\
& Q_{12}=Q_{21}=\frac{\nu E}{1-\nu^{2}}, \\
& Q_{66}=\frac{E}{2(1+\nu)},
\end{aligned}
$$

where $E$ and $v$ are Young's modulus and Poisson's ratio, respectively.

From the theory of elasticity, the strain energy equation in the shells can be expressed as

$$
\begin{aligned}
U_{\xi}^{i} & =\frac{1}{2} \int_{V}\left(1+\frac{z_{\xi}}{R_{\varphi_{\xi}}}\right)\left(1+\frac{z_{\xi}}{R_{\theta_{\xi}}}\right) \\
& \cdot\left(\sigma_{\varphi_{\xi}}^{i} e_{\varphi_{\xi}}^{i}+\sigma_{\theta_{\xi}}^{i} e_{\theta_{\xi}}^{i}+\sigma_{\varphi \theta_{\xi}}^{i} \gamma_{\varphi \theta_{\xi}}^{i}\right) A B d \varphi_{\xi} d \theta_{\xi} d z_{\xi} .
\end{aligned}
$$


In the case of the thin shells, we can simplify some of the terms in the energy equation, and the energy equation is rearranged through a mathematical process:

$$
\begin{aligned}
U_{\xi}^{i}= & \frac{E h}{2\left(1-v^{2}\right)} \iint\left[Q_{\xi, 0}^{i}+\frac{h^{2}}{12}\left(Q_{\xi, 1}^{i}+Q_{\xi, 2}^{i}\right)\right] \\
& \cdot A B d \varphi_{\xi} d \theta_{\xi},
\end{aligned}
$$

where

$$
\begin{aligned}
Q_{\xi, 0}^{i}= & \left(\varepsilon_{\varphi_{\xi}}^{i}\right)^{2}+\left(\varepsilon_{\theta_{\xi}}^{i}\right)^{2}+2 v \varepsilon_{\varphi_{\xi}}^{i} \varepsilon_{\theta_{\xi}}^{i}+\frac{1-\nu}{2}\left(\varepsilon_{\varphi \theta_{\xi}}^{i}\right)^{2}, \\
Q_{\xi, 1}^{i}= & \left(k_{\varphi_{\xi}}^{i}\right)^{2}+\left(k_{\theta_{\xi}}^{i}\right)^{2}+2 v k_{\varphi_{\xi}}^{i} k_{\theta_{\xi}}^{i}+\frac{1-v}{2}\left(k_{\varphi \theta_{\xi}}^{i}\right)^{2}, \\
Q_{\xi, 1}^{i}= & -2\left(\frac{1}{R_{\varphi_{\xi}}}-\frac{1}{R_{\theta_{\xi}}}\right)\left(\varepsilon_{\varphi_{\xi}}^{i} k_{\varphi_{\xi}}^{i}-\varepsilon_{\theta_{\xi}}^{i} k_{\theta_{\xi}}^{i}\right) \\
& -\frac{1-v}{2}\left(\frac{1}{R_{\varphi_{\xi}}}+\frac{1}{R_{\theta_{\xi}}}\right) \varepsilon_{\varphi_{\theta_{\xi}}}^{i} k_{\varphi_{\theta_{\xi}}}^{i} \\
& +\left(\frac{1}{R_{\varphi_{\xi}}}-\frac{1}{R_{\theta_{\xi}}}\right)\left(\frac{\left(\varepsilon_{\varphi_{\xi}}^{i}\right)^{2}}{R_{\varphi_{\xi}}}-\frac{\left(\varepsilon_{\theta_{\xi}}^{i}\right.}{R_{\theta_{\xi}}}\right)^{2} \\
& +\frac{1-\nu}{2}\left(\frac{1}{R_{\varphi_{\xi}}^{2}}+\frac{1}{R_{\theta_{\xi}}^{2}}-\frac{1}{R_{\varphi_{\xi}} R_{\theta_{\xi}}}\right)\left(\varepsilon_{\varphi \theta_{\xi}}^{i}\right)^{2} .
\end{aligned}
$$

And the kinetic energy of the shell components can be defined as

$$
T_{\xi}^{i}=\frac{\rho h}{2} \iint\left[\left(\dot{u}_{\xi}^{i}\right)^{2}+\left(\dot{v}_{\xi}^{i}\right)^{2}+\left(\dot{w}_{\xi}^{i}\right)^{2}\right] A B d \varphi_{\xi} d \theta_{\xi} .
$$

In order to investigate the forced vibration of the airtight cylindrical vessels under point loads, it is assumed that the external unit force acts on the middle surface of the vessel in the meridional, circumferential, and the normal directions, respectively. The virtual work by the external forces can be written as

$$
\begin{gathered}
W_{\xi}^{i}=\iint\left(f_{u} u_{\xi}^{i}+f_{v} v_{\xi}^{i}+f_{w} w_{\xi}^{i}\right) \delta\left(\varphi_{\xi}-\varphi_{f}\right) \\
\cdot \delta\left(\theta_{\xi}-\theta_{f}\right) e^{i \omega t} A B d \varphi_{\xi} d \theta_{\xi},
\end{gathered}
$$

where $\delta$ is the Dirac function and $f_{u}, f_{v}$, and $f_{w}$ are the external force in the meridional, circumferential, and the normal directions, respectively.

2.3. Continuity Conditions of the Airtight Cylindrical Vessel. In this analysis, the continuity conditions of the airtight cylindrical vessel structures with doubly curved revolution shells are modeled by the penalty parameters, which are characterized by artificial spring stiffness. The penalty parameter permits the flexible selection of the admissible displacement functions of the airtight cylindrical vessels, and the appropriate value of the penalty parameter ensures fast convergence of the accurate solution. Thus, the potential energy stored in the connective springs at the junction between the doubly curved shells and the cylindrical shell can be described as

$$
\begin{aligned}
U_{c} & =\frac{1}{2} \int_{0}^{2 \pi}\left\{k_{u}\left[u_{c}-\left(u_{l} \sin \varphi_{l}-w_{l} \cos \varphi_{l}\right)\right]^{2}\right. \\
& +k_{v}\left(v_{c}-v_{l}\right)^{2}+k_{w}\left[w_{c}-\left(u_{l} \cos \varphi_{l}+w_{l} \sin \varphi_{l}\right)\right]^{2} \\
& \left.+k_{r}\left[\frac{\partial w_{c}}{\partial \varphi_{c}}+\frac{\left(u_{l}-\partial w_{l} / \partial \varphi_{l}\right)}{R_{\varphi_{l}}}\right]^{2}\right\} \\
& \cdot \int_{0}^{2 \pi}\left\{k_{u}\left[u_{c}+\left(u_{r} \sin \varphi_{r}-w_{r} \cos \varphi_{r}\right)\right]^{2}\right. \\
& +k_{v}\left(v_{c}-v_{r}\right)^{2}+k_{w}\left[w_{c}-\left(u_{r} \cos \varphi_{r}+w_{r} \sin \varphi_{r}\right)\right]^{2} \\
& \left.+k_{r}\left[\frac{\partial w_{c}}{\partial \varphi_{c}}+\frac{\left(u_{r}-\partial w_{r} / \partial \varphi_{r}\right)}{R_{\varphi_{r}}}\right]^{2}\right\}_{\varphi_{c}, \varphi_{r}} B d \theta_{c},
\end{aligned}
$$

where $k_{u}, k_{v}, k_{w}$, and $k_{r}$ denote the stiffness of the translational and rotational springs between the shell components, respectively.

If each shell component is further subdivided into shell segments, the potential energy stored in the connective springs of two adjacent shell segments can be described as follows:

$$
\begin{aligned}
U_{s, l}^{i} & =\frac{1}{2} \int_{0}^{2 \pi}\left\{k_{u}\left(u_{l}^{i}-u_{l}^{i+1}\right)^{2}+k_{v}\left(v_{l}^{i}-v_{l}^{i+1}\right)^{2}\right. \\
& +k_{w}\left(w_{l}^{i}-w_{l}^{i+1}\right)^{2} \\
& \left.+\frac{k_{r}}{R_{\varphi_{l}}^{2}}\left[\left(u_{l}^{i}-\frac{\partial w_{l}^{i}}{\partial \varphi_{l}}\right)-\left(u_{l}^{i+1}-\frac{\partial w_{l}^{i+1}}{\partial \varphi_{l}}\right)\right]^{2}\right\}_{i, i+1} \\
& \cdot B d \theta_{l}, \\
U_{s, c}^{i} & =\frac{1}{2} \int_{0}^{2 \pi}\left\{k_{u}\left(u_{c}^{i}-u_{c}^{i+1}\right)^{2}+k_{v}\left(v_{c}^{i}-v_{c}^{i+1}\right)^{2}\right. \\
+ & \left.k_{w}\left(w_{c}^{i}-w_{c}^{i+1}\right)^{2}+k_{r}\left(\frac{\partial w_{c}^{i}}{\partial \varphi_{c}}-\frac{\partial w_{c}^{i+1}}{\partial \varphi_{c}}\right)^{2}\right\}_{i, i+1} \\
\cdot & B d \theta_{c}, \\
U_{s, r}^{i} & =\frac{1}{2} \int_{0}^{2 \pi}\left\{k_{u}\left(u_{r}^{i}-u_{r}^{i+1}\right)^{2}+k_{v}\left(v_{r}^{i}-v_{r}^{i+1}\right)^{2}\right. \\
+ & k_{w}\left(w_{r}^{i}-w_{r}^{i+1}\right)^{2} \\
+ & \left.\frac{k_{r}}{R_{\varphi_{r}}^{2}}\left[\left(u_{r}^{i}-\frac{\partial w_{r}^{i}}{\partial \varphi_{r}}\right)-\left(u_{r}^{i+1}-\frac{\partial w_{r}^{i+1}}{\partial \varphi_{r}}\right)\right]^{2}\right\} \\
\cdot & B d \theta_{r},
\end{aligned}
$$


where $U_{s, l}^{i}, U_{s, c}^{i}$, and $U_{s, r}^{i}$ represent the potential energy stored in the connective springs of the shell segments at the left, cylindrical, and right shell, respectively. And the superscripts, $i$ and $i+1$, represent the $i$ th and $(i+1)$ th shell segments.

Therefore, the total potential energy reflecting the connective conditions can be expressed as

$$
U_{B C}=U_{c}+\sum_{i=1}^{N_{l}-1} U_{s, l}^{i}+\sum_{i=1}^{N_{c}-1} U_{s, c}^{i}+\sum_{i=1}^{N_{r}-1} U_{s, r}^{i}
$$

where $N_{l}, N_{c}$, and $N_{r}$ are the number of segments divided in the each shell components.

2.4. Unified Solution and Solution Procedure. The selection of an admissible displacement function is an important factor in guaranteeing a stable convergence and accuracy of the solution. In the handling of the continuity conditions, the use of spring stiffness, which can be seen as penalty parameters, makes the selection of admissible functions very flexible [3747]. Subscript $i$ is omitted here for the sake of brevity. In this work, the admissible displacement functions of shell segments are generally extended to the Jacobi orthogonal polynomials and Fourier series, regardless of shape of the shell components. As we know, classical Jacobi polynomials [48] are defined on the interval of $\phi \in[-1,1]$ and the recurrence formula of the classical Jacobi polynomials $P_{i}^{(\alpha, \beta)}(\phi)$ of degree $i$ is given by

$$
\begin{aligned}
& P_{0}^{(\alpha, \beta)}(\phi)=1, \\
& P_{1}^{(\alpha, \beta)}(\phi)=\frac{\alpha+\beta+2}{2} \phi-\frac{\alpha-\beta}{2}, \\
& P_{i}^{(\alpha, \beta)}(\phi) \\
& \quad=\frac{(\alpha+\beta+2 i-1)\left\{\alpha^{2}-\beta^{2}+\phi(\alpha+\beta+2 i)(\alpha+\beta+2 i-2)\right\}}{2 i(\alpha+\beta+i)(\alpha+\beta+2 i-2)} \\
& \quad \cdot P_{i-1}^{(\alpha, \beta)}(\phi)-\frac{(\alpha+i-1)(\beta+i-1)(\alpha+\beta+2 i)}{i(\alpha+\beta+i)(\alpha+\beta+2 i-2)} P_{i-2}^{(\alpha, \beta)}(\phi),
\end{aligned}
$$

where $\alpha, \beta>-1$ and $i=2,3, \ldots$.

The orthogonality condition of classical Jacobi polynomials is

$$
\int_{-1}^{1} P_{j}^{(\alpha, \beta)}(\phi) P_{k}^{(\alpha, \beta)}(\phi) w^{(\alpha, \beta)}(\phi) d \phi=h_{k},
$$

where $w^{(\alpha, \beta)}(x)=(1-x)^{\alpha}(1+x)^{\beta}$ and

$$
\begin{aligned}
& h_{k} \\
& = \begin{cases}\frac{2^{\alpha+\beta+1}}{2 k+\alpha+\beta+1} \frac{\Gamma(k+\alpha+1) \Gamma(k+\beta+1)}{\Gamma(k+\alpha+\beta+1) k !}, & j=k \\
0, & j \neq k .\end{cases}
\end{aligned}
$$

The Jacoby polynomials are generalized orthonormal polynomials containing some orthonormal polynomials such as Legendre, Chebyshev, and Gegenbauer polynomials. For example, the choice $\alpha=\beta=-1 / 2$ yields the Chebyshev polynomials of the first kind, while choosing $\alpha=\beta=1 / 2$ gives the Chebyshev polynomials of the second kind. Also the choice $\alpha=\beta=0$ yields the Legendre polynomials, while choosing $\alpha=\beta$ gives the Gegenbauer polynomials. Thus, the selection of an admissible displacement function for the airtight cylindrical vessels is more generalized by the Jacobi polynomials.

The displacement functions of the shell segments can be written in the forms

$$
\begin{gathered}
u_{\xi}=\sum_{m=0}^{M} \sum_{n=0}^{N} U_{m n, \xi} P_{m}^{(\alpha, \beta)}(\phi) \cos \left(n \theta_{\xi}\right) e^{i \omega t}, \\
v_{\xi}=\sum_{m=0}^{M} \sum_{n=0}^{N} V_{m n, \xi} P_{m}^{(\alpha, \beta)}(\phi) \sin \left(n \theta_{\xi}\right) e^{i \omega t}, \\
w_{\xi}=\sum_{m=0}^{M} \sum_{n=0}^{N} W_{m n, \xi} P_{m}^{(\alpha, \beta)}(\phi) \cos \left(n \theta_{\xi}\right) e^{i \omega t},
\end{gathered}
$$

where $U_{m n, \xi}, V_{m n, \xi}$, and $W_{m n, \xi}$ are unknown coefficients of the Jacobi polynomials that you want to obtain; $P_{m}^{(\alpha, \beta)}(\phi)$ is the $m$ th order Jacobi polynomial for the displacement function in the meridional direction; $\omega$ is an angular frequency; $t$ denotes time. The nonnegative integers $M$ and $N$ represent the highest degrees taken in the Jacobi polynomials and the Fourier series; the positive integer $n$ represents the mode circumference wave number. The subscript $\xi(\xi=l, c, r)$ means the left, cylindrical, and right shells, respectively. The classical Jacobi polynomials are complete and orthogonal polynomials defined on the interval of $\phi \in[-1,1]$. Thus, a linear transformation rule is introduced for coordinate conversion from interval $\varphi \in\left[\varphi_{\xi}^{i}, \varphi_{\xi}^{i+1}\right]$ of the $i$ th shell segment to interval $\phi(\phi \in[-1,1])$ of the Jacobi polynomials, that is, $\varphi_{\xi}=\left[\left(\varphi_{\xi}^{i+1}-\varphi_{\xi}^{i}\right) / 2\right] \phi+\left(\varphi_{\xi}^{i+1}+\varphi_{\xi}^{i}\right) / 2$.

The total Lagrangian energy functions $(L)$ of the airtight cylindrical vessels with doubly curved shells of revolution can be written in the forms

$$
\begin{aligned}
L= & \sum_{i=1}^{N_{l}}\left(T_{l}^{i}-U_{l}^{i}\right)+\sum_{i=1}^{N_{c}}\left(T_{c}^{i}-U_{c}^{i}\right)+\sum_{i=1}^{N_{r}}\left(T_{r}^{i}-U_{r}^{i}\right) \\
& -U_{B C}+W_{\xi}^{i},
\end{aligned}
$$

where $U_{B C}$ represent total potential energy reflecting the connective conditions and $W_{\xi}^{i}$ represent the virtual work by the external forces.

The total Lagrangian energy function is minimized with respect to the unknown coefficients by using the RayleighRitz method.

$$
\begin{aligned}
\frac{\partial L}{\partial \vartheta} & =0 \\
\vartheta & =U_{m n, \xi}, V_{m n, \xi}, W_{m n, \xi}, \\
\left(\mathbf{K}-\omega^{2} \mathbf{M}\right) \mathbf{X} & =\mathbf{F}
\end{aligned}
$$

where $\mathbf{K}$ and $\mathbf{M}$ are the stiffness and mass matrix of the airtight cylindrical vessel, respectively. And $\mathbf{X}$ and $\mathbf{F}$ represent the coefficient vector and external force vector. If $\mathbf{F}$ is a 


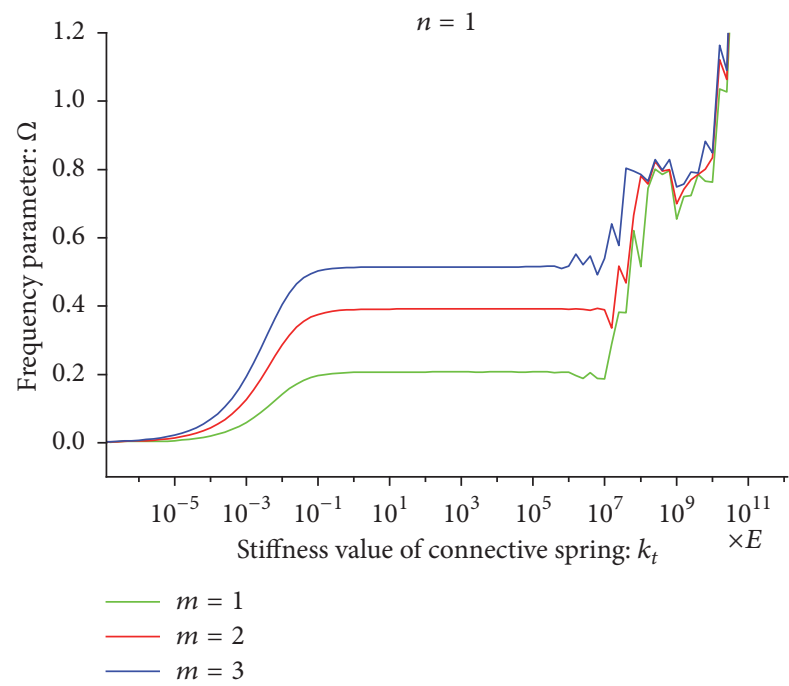

(a)

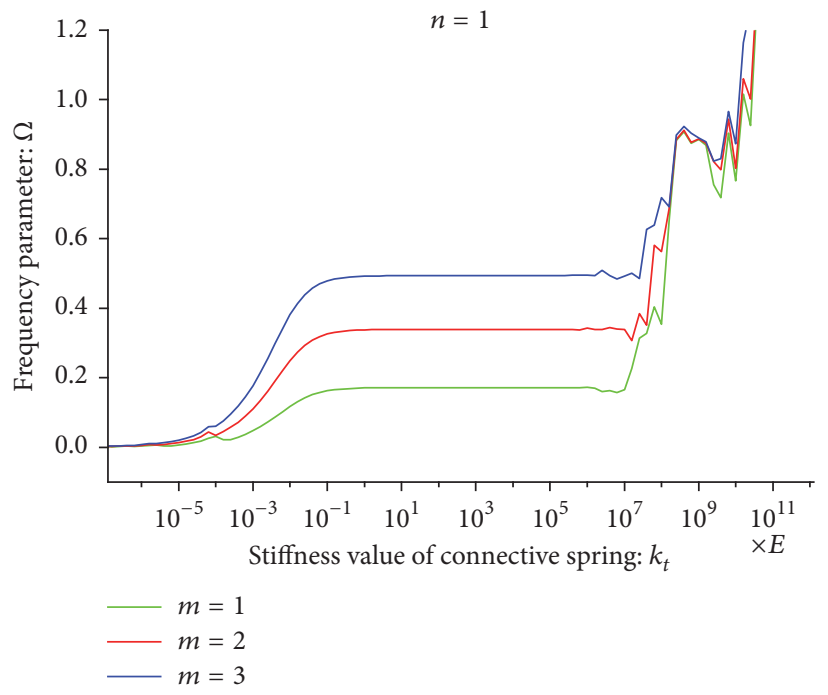

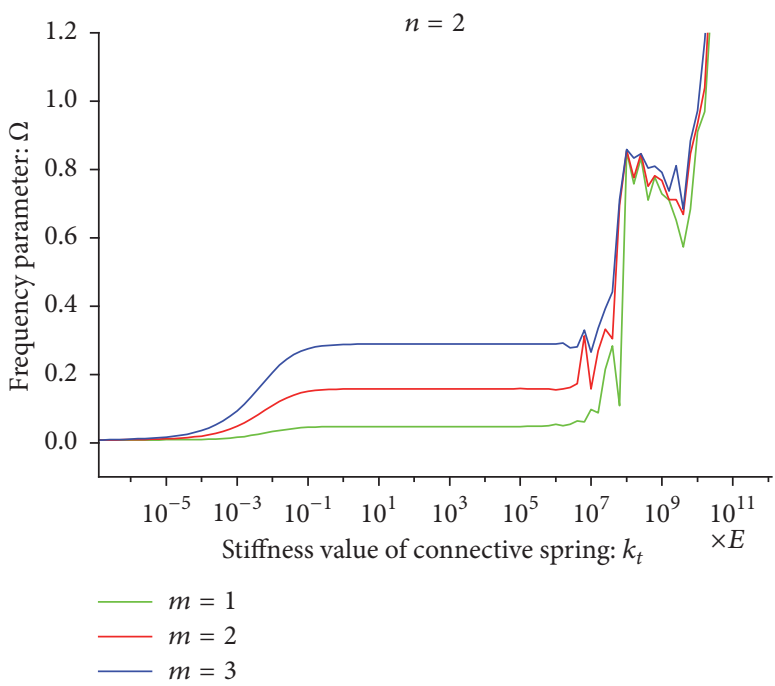

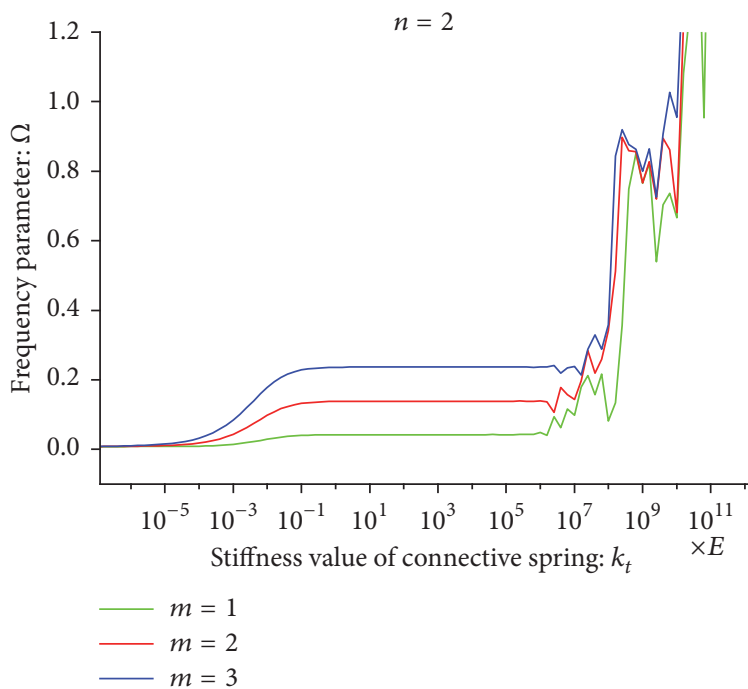

(b)

FIGURE 2: The convergence characteristics of nondimensionless frequency parameters for two vessels: (a) ECE vessel; (b) PCE vessel.

zero vector, the above equation is the free vibration analysis equation, and if it is not a zero vector, it is the forced vibration analysis equation. A detailed description of (21) is given in Appendix.

\section{Numerical Results and Discussions}

3.1. Convergence and Comparison Studies. In this section, the reliability and accuracy of this method are verified by comparing the results of the method with those of the previous literature or the finite element method (FEM). From the mathematical point of view, unlike the domain decomposition method which uses the Lagrange multipliers and the weight residual least squares method [35], when the penalty parameters is defined as a very high value the solution of the proposed method may not converge [38-40]. And it is necessary to emphasize that the research will focus on the elastic deformation rather than rigid deformation. So some zero eigenvalue obtained in the solution will be ignored in the following research. The convergence characteristics of the nondimensionless frequency parameters for different spring stiffness values in the ECE and the PCE vessels are shown in Figure 2. Where the material parameters and the geometric dimensions of the elliptical-cylindrical-elliptical and the paraboloidal-cylindrical-elliptical vessels are as follows:

ECE vessel:

$$
\begin{aligned}
& E=211 \mathrm{Gpa}, \rho=7800 \mathrm{~kg} / \mathrm{m}^{3}, v=0.3, R_{c}=1 \mathrm{~m}, \\
& L_{c}=6 \mathrm{~m}, h=0.01 \mathrm{~m}, L_{l}=0.5 \mathrm{~m}, L_{r}=0.5 \mathrm{~m} .
\end{aligned}
$$

PCE vessel:

$$
\begin{aligned}
& E=211 \mathrm{Gpa}, \rho=7800 \mathrm{~kg} / \mathrm{m}^{3}, \nu=0.3, R_{c}=1 \mathrm{~m}, \\
& L_{c}=6 \mathrm{~m}, h=0.01 \mathrm{~m}, L_{l}=2 \mathrm{~m}, L_{r}=0.5 \mathrm{~m} .
\end{aligned}
$$

In this analysis, the number of segments of the two structures is taken as $N_{l}=4, N_{c}=8$, and $N_{r}=4$, 
TABLE 1: Frequency parameter $\Omega=\omega R_{c}^{2} \sqrt{\rho(1-\nu)^{2} / E}$ of a spherical-cylindrical-spherical shell.

\begin{tabular}{|c|c|c|c|c|c|c|c|c|}
\hline \multicolumn{2}{|c|}{ Mode number } & \multicolumn{5}{|c|}{ Number of the segments $\left(N_{l}=N_{c}=N_{r}\right)$} & \multirow{2}{*}{ Shi et al. [37] } & \multirow{2}{*}{ ANSYS [37] } \\
\hline$n$ & $m$ & 2 & 4 & 6 & 8 & 10 & & \\
\hline \multirow{7}{*}{0} & 1 & 0.04872 & 0.04872 & 0.04872 & 0.04872 & 0.04872 & 0.04872 & 0.04860 \\
\hline & 2 & 0.06094 & 0.06092 & 0.06092 & 0.06092 & 0.06092 & 0.06101 & 0.06085 \\
\hline & 3 & 0.08434 & 0.08432 & 0.08432 & 0.08432 & 0.08432 & 0.08455 & 0.08409 \\
\hline & 4 & 0.09565 & 0.09565 & 0.09565 & 0.09565 & 0.09565 & 0.09566 & 0.09567 \\
\hline & 5 & 0.09574 & 0.09574 & 0.09574 & 0.09574 & 0.09574 & 0.09581 & 0.09568 \\
\hline & 6 & 0.10132 & 0.10132 & 0.10132 & 0.10132 & 0.10132 & 0.10140 & 0.10118 \\
\hline & 7 & 0.10360 & 0.10359 & 0.10359 & 0.10360 & 0.10359 & 0.10373 & 0.10334 \\
\hline \multirow{7}{*}{1} & 1 & 0.04175 & 0.04174 & 0.04174 & 0.04175 & 0.04173 & 0.04180 & 0.04165 \\
\hline & 2 & 0.06391 & 0.06390 & 0.06390 & 0.06390 & 0.06390 & 0.06399 & 0.06384 \\
\hline & 3 & 0.07743 & 0.07743 & 0.07742 & 0.07742 & 0.07742 & 0.07751 & 0.07732 \\
\hline & 4 & 0.08827 & 0.08825 & 0.08825 & 0.08825 & 0.08825 & 0.08850 & 0.08801 \\
\hline & 5 & 0.09016 & 0.09016 & 0.09016 & 0.09016 & 0.09016 & 0.09014 & 0.09015 \\
\hline & 6 & 0.09626 & 0.09626 & 0.09626 & 0.09625 & 0.09625 & 0.09623 & 0.09622 \\
\hline & 7 & 0.10067 & 0.10067 & 0.10067 & 0.10066 & 0.10066 & 0.10104 & 0.10019 \\
\hline \multirow{7}{*}{2} & 1 & 0.01312 & 0.01312 & 0.01312 & 0.01312 & 0.01312 & 0.01327 & 0.01300 \\
\hline & 2 & 0.03779 & 0.03777 & 0.03777 & 0.03777 & 0.03777 & 0.03800 & 0.03760 \\
\hline & 3 & 0.06021 & 0.06020 & 0.06020 & 0.06020 & 0.06020 & 0.06042 & 0.06000 \\
\hline & 4 & 0.07636 & 0.07635 & 0.07635 & 0.07635 & 0.07635 & 0.07649 & 0.07620 \\
\hline & 5 & 0.08683 & 0.08683 & 0.08683 & 0.08683 & 0.08683 & 0.08689 & 0.08680 \\
\hline & 6 & 0.09321 & 0.09321 & 0.09321 & 0.09321 & 0.09321 & 0.09328 & 0.09310 \\
\hline & 7 & 0.09670 & 0.09669 & 0.09669 & 0.09669 & 0.09669 & 0.09684 & 0.09660 \\
\hline
\end{tabular}

the parameters of the Jacobi polynomials are taken as $\alpha=$ $-0.5, \beta=-0.5$, and the nondimensionless frequency parameter is defined as $\Omega=\omega R_{c} \sqrt{\rho(1-\nu)^{2} / E}$.

As can be seen in Figure 2, it can be seen that the connection spring stiffness value ensures stable convergence within the range of $k_{t}=10^{0} E \sim 10^{6} \mathrm{E}$. Therefore, unless otherwise noted, in this paper, it is assumed that the connective spring stiffness is the same (i.e., $\left.k_{t}=10^{3} E,(t=u, v, w)\right)$.

Next, in order to verify the accuracy of the presented method, free vibration of a spherical-cylindrical-spherical shell having the same material characteristics and structural dimensions as those of the previous literature is considered. Frequency convergence results for the shell segments $\left(N_{l}, N_{c}, N_{r}\right)$ of the spherical-cylindrical-spherical shell are shown in Table 1 and the results are compared with those of the previous literature [37].

In Table 1, it can be seen that the frequency quickly converges to correct solution with the number of shell segments increasing. However, for simplicity the calculation, the number of shell segments is limited to eight or less in this paper. The percentage error of the solution for the Jacobi parameters $\alpha$ and $\beta$ in the elliptical-cylindrical-elliptical shell are pointed out in Figure 3.

As shown in Figure 3, it can be seen that the change of the Jacobi parameters $\alpha$ and $\beta$ does not have a great influence on the frequency convergence and the maximum error does not exceed $5 \cdot 10^{-4}$. This means that not only the Chebyshev, Legendre, Bernstein, and Gegenbauer polynomials but also the generalized Jacobi polynomial can be used. In Table 2, the comparison between the results of the ECE and the PCE vessels pointed out above and the finite element method (FEM) are shown. In here, the ECE vessel used the S4R model and the 19433 element, and the PCE vessel used the S4R model and the 19389 element of the ABAQUS.

The validity and accuracy of the presented method were verified by comparisons with the above results. For the sake of understanding, unless otherwise stated, the parameters of the structure and the material properties in the subsequent study are taken as follows:

$$
\begin{aligned}
& E=211 \mathrm{Gpa}, \rho=7800 \mathrm{~kg} / \mathrm{m}^{3}, v=0.3, M=N= \\
& 8, N_{l}=4, N_{c}=8, N_{r}=4, \alpha=0, \beta=0, R_{c}= \\
& 1 \mathrm{~m}, L_{c}=6 \mathrm{~m}, h=0.01 \mathrm{~m}, L_{r}=0.5 \mathrm{~m} .
\end{aligned}
$$

Nondimensional frequency parameter is defined as $\Omega=$ $\omega R_{c} \sqrt{\rho(1-v)^{2} / E}$.

3.2. Free Vibration of the Airtight Cylindrical Vessel. In this section, the study on free vibration analysis of elliptical-cylindrical-elliptical vessel and paraboloidalcylindrical-elliptical vessel is performed. The change in frequency parameters for the axial wave number $m$ and the circumferential wave number $n$ in the ECE vessel and the PCE vessel used in Figure 2 is shown in Figure 4.

As shown in Figure 4, when $n=3$, the lowest frequency of the two structures is shown, and, as $n$ increases, the frequency parameters increased and the difference between 


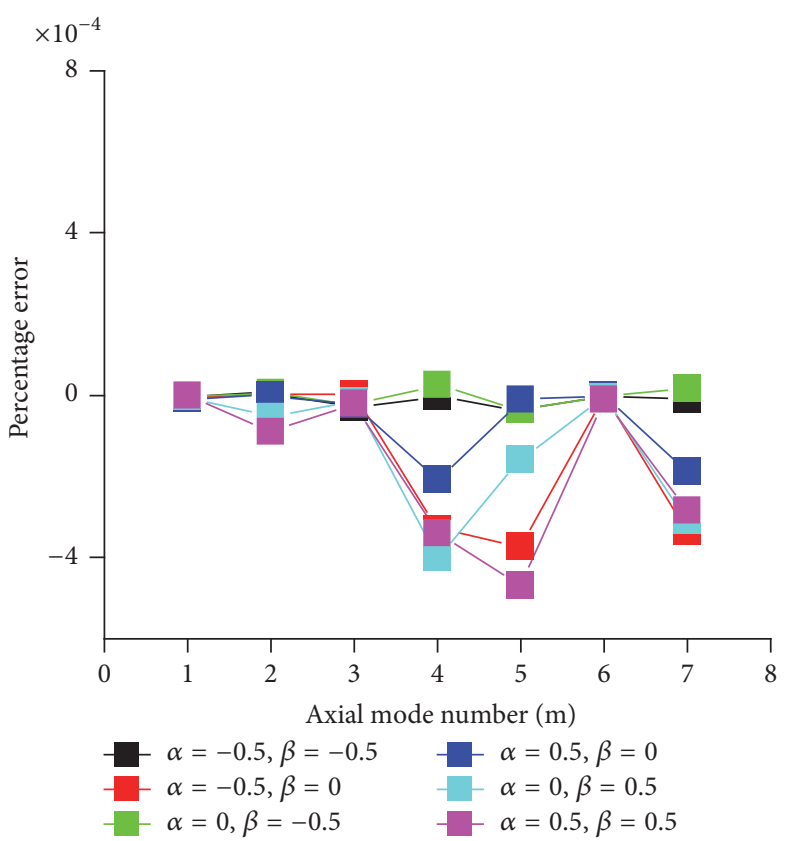

(a) $n=0$

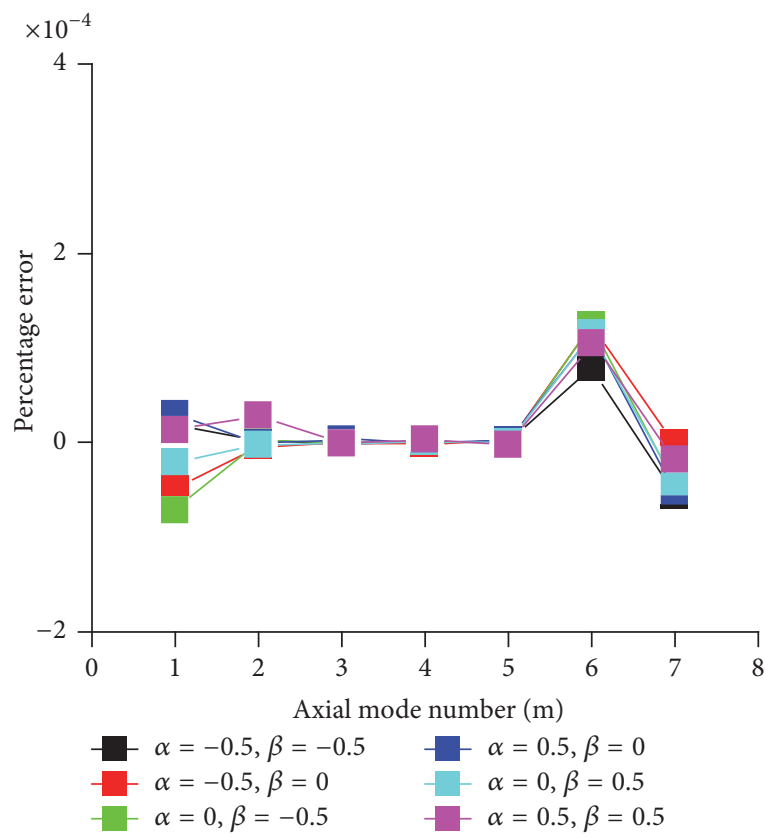

(c) $n=2$

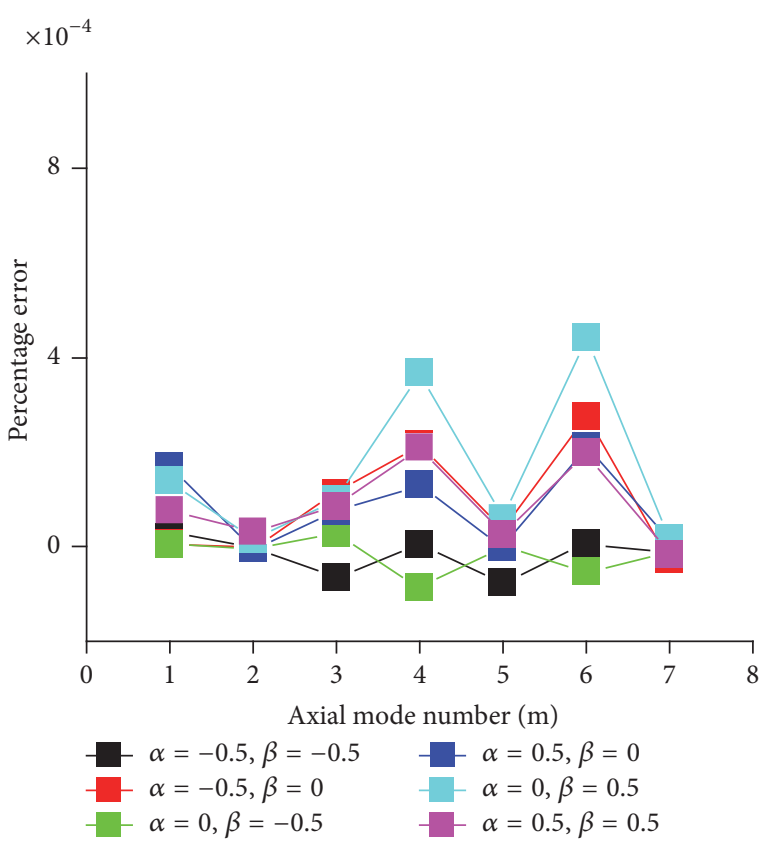

(b) $n=1$

FIgURE 3: Percentage error of the frequency for the Jacobi parameters and in the elliptical-cylindrical-elliptical shell.

the frequency parameters is decreased. Table 3 shows the frequency parameters according to the ratio between the semimajor axis and the semiminor axis of the right ellipsoid in the elliptical-cylindrical-elliptical vessel, and Table 4 shows the frequency parameters according to the ratio between the maximum radius and the length of the paraboloid in the paraboloidal-cylindrical-elliptical vessel.

From Tables 3 and 4, it can be seen that the frequency parameters of the structures are significantly reduced as two ratios gradually increase. In addition, to facilitate the reader's understanding, some mode shapes of the ECE vessel and PCE vessel are shown in Figure 5.

3.3. Forced Vibration of the Airtight Cylindrical Vessels. Analyzing the forced vibration of a vessel subjected to a point load is an important factor in studying the vibration response characteristics of the vessel. In this study, the forced vibration of the structure is investigated assuming that the axial unit force $f_{u}\left(f_{u}=1 N\right)$ and the radial unit force $f_{w}\left(f_{w}=1 N\right)$ act separately on the vessels used in Figure 1. 
TABLE 2: Comparison of some frequency parameters $\Omega=\omega R_{c} \sqrt{\rho(1-v)^{2} / E}$ for the ECE vessel and PCE vessel $\left(N_{l}=4, N_{c}=8, N_{r}=4\right.$, $\alpha=-0.5$, and $\beta=-0.5)$.

\begin{tabular}{|c|c|c|c|c|c|}
\hline \multicolumn{2}{|c|}{ Mode number } & \multicolumn{2}{|c|}{ ECE vessel } & \multicolumn{2}{|c|}{ PCE vessel } \\
\hline$n$ & $m$ & Present & FEM & Present & FEM \\
\hline \multirow{5}{*}{0} & 1 & 0.27209 & 0.27203 & 0.25524 & 0.25520 \\
\hline & 2 & 0.36257 & 0.36328 & 0.34346 & 0.34549 \\
\hline & 3 & 0.45512 & 0.45653 & 0.46885 & 0.47130 \\
\hline & 4 & 0.49345 & 0.49265 & 0.50378 & 0.50361 \\
\hline & 5 & 0.52093 & 0.52117 & 0.52362 & 0.52489 \\
\hline \multirow{5}{*}{1} & 1 & 0.20694 & 0.20708 & 0.06650 & 0.06619 \\
\hline & 2 & 0.39073 & 0.39132 & 0.19918 & 0.19861 \\
\hline & 3 & 0.51455 & 0.51439 & 0.34286 & 0.34208 \\
\hline & 4 & 0.51863 & 0.51785 & 0.47787 & 0.47683 \\
\hline & 5 & 0.55112 & 0.55172 & 0.58726 & 0.58442 \\
\hline \multirow{5}{*}{2} & 1 & 0.04723 & 0.04754 & 0.04193 & 0.04231 \\
\hline & 2 & 0.15759 & 0.15815 & 0.13737 & 0.13842 \\
\hline & 3 & 0.28803 & 0.28890 & 0.23527 & 0.23682 \\
\hline & 4 & 0.41272 & 0.41382 & 0.31923 & 0.31989 \\
\hline & 5 & 0.52104 & 0.52222 & 0.41943 & 0.42089 \\
\hline
\end{tabular}

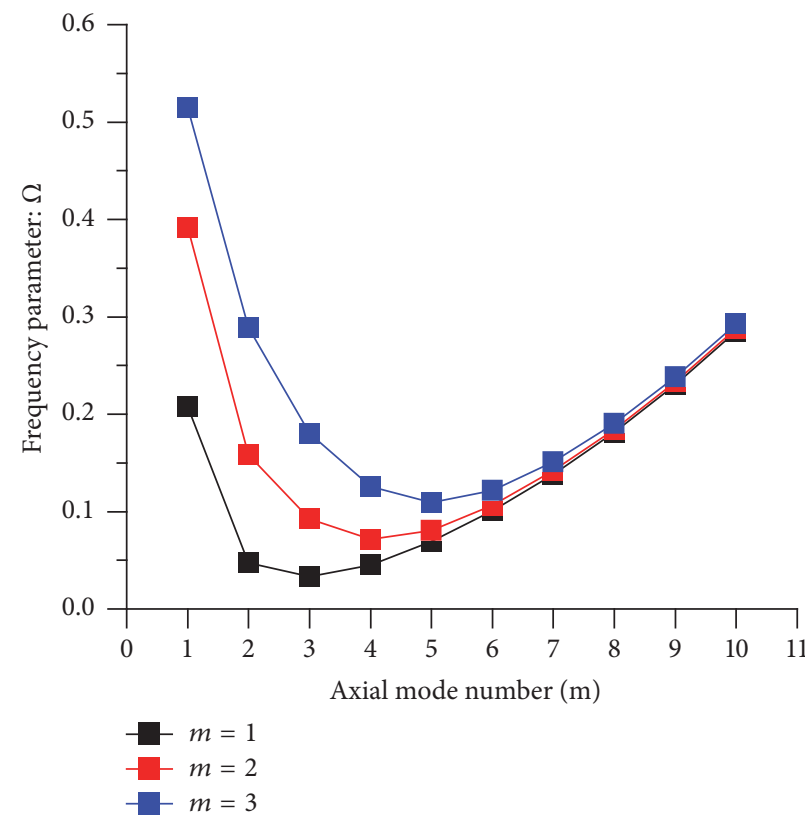

(a)

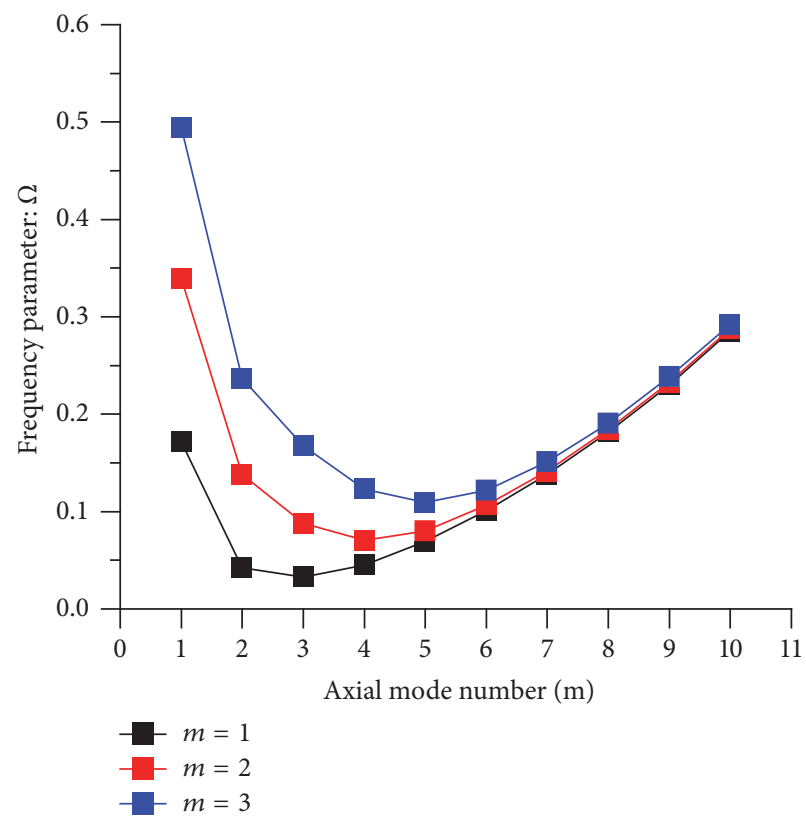

(b)

FIGURE 4: The change in frequency parameters for the axial and the circumferential wave number, (a) ECE vessel, (b) PCE vessel.

And the structural damping is realized by using a complex modulus $E_{d}=(1+i \eta) E$ instead of the original Young's modulus $E$, where $\eta(\eta=0.002)$ is defined as the damping of the vessel structures [33]. Forced vibration analysis is investigated between the frequency domain $20 \sim 500 \mathrm{~Hz}$ and the calculation frequency step is adopted as $0.2 \mathrm{~Hz}$. The fourpoint coordinate systems $D_{1} \sim D_{4}$ are set in the ECE vessel and the PCE vessel. In the ECE vessel and the PCE vessel, the radial displacements of point $D_{3}$ when the radial force $f_{w}\left(f_{w}=1 N\right)$ acts on point $D_{2}$ are shown in Figure 5 , and this is compared with FEM results (ECE vessel; S4R model, 19433 element, PCE vessel; S4R model, 19389 element).

The comparisons of Figures 5 and 6 show that the results of the presented method are in good agreement with those 
TABLE 3: The frequency parameters $\Omega$ of the ECE vessel.

\begin{tabular}{|c|c|c|c|c|c|c|c|}
\hline \multicolumn{2}{|c|}{ Mode number } & \multicolumn{6}{|c|}{$b_{l} / a_{l}$} \\
\hline$n$ & $m$ & 0.25 & 0.75 & 1 & 1.5 & 2 & 4 \\
\hline \multirow{5}{*}{1} & 1 & 0.21294 & 0.20016 & 0.19303 & 0.17892 & 0.16554 & 0.12115 \\
\hline & 2 & 0.28211 & 0.38297 & 0.37333 & 0.35314 & 0.33271 & 0.25608 \\
\hline & 3 & 0.34942 & 0.51601 & 0.51518 & 0.50953 & 0.49062 & 0.38977 \\
\hline & 4 & 0.40583 & 0.54892 & 0.54102 & 0.52504 & 0.51931 & 0.50164 \\
\hline & 5 & 0.48499 & 0.57600 & 0.57432 & 0.57110 & 0.56761 & 0.52089 \\
\hline \multirow{5}{*}{2} & 1 & 0.04915 & 0.04599 & 0.04449 & 0.04165 & 0.03901 & 0.03023 \\
\hline & 2 & 0.16195 & 0.15409 & 0.14986 & 0.14118 & 0.13230 & 0.09610 \\
\hline & 3 & 0.29253 & 0.28359 & 0.27743 & 0.26314 & 0.24612 & 0.16737 \\
\hline & 4 & 0.33434 & 0.40855 & 0.40148 & 0.38216 & 0.35420 & 0.24808 \\
\hline & 5 & 0.41344 & 0.51765 & 0.51034 & 0.48569 & 0.44557 & 0.33182 \\
\hline \multirow{5}{*}{3} & 1 & 0.03379 & 0.03286 & 0.03242 & 0.03159 & 0.03083 & 0.02832 \\
\hline & 2 & 0.09443 & 0.09063 & 0.08874 & 0.08499 & 0.08126 & 0.06605 \\
\hline & 3 & 0.18303 & 0.17682 & 0.17348 & 0.16637 & 0.15855 & 0.11518 \\
\hline & 4 & 0.27993 & 0.27265 & 0.26824 & 0.25796 & 0.24463 & 0.16372 \\
\hline & 5 & 0.37353 & 0.36688 & 0.36189 & 0.34871 & 0.32661 & 0.22331 \\
\hline
\end{tabular}

TABLE 4: The frequency parameters $\Omega$ of the PCE vessel.

\begin{tabular}{|c|c|c|c|c|c|c|c|}
\hline \multicolumn{2}{|c|}{ Mode number } & \multicolumn{6}{|c|}{$L_{l} / R_{c}$} \\
\hline$n$ & $m$ & 0.5 & 1 & 3 & 4 & 5 & 6 \\
\hline \multirow{5}{*}{1} & 1 & 0.21003 & 0.19808 & 0.14626 & 0.12231 & 0.09940 & 0.07730 \\
\hline & 2 & 0.39050 & 0.37759 & 0.29602 & 0.25246 & 0.21458 & 0.18308 \\
\hline & 3 & 0.51339 & 0.51519 & 0.43596 & 0.38056 & 0.33747 & 0.30398 \\
\hline & 4 & 0.52518 & 0.54030 & 0.51637 & 0.50212 & 0.46736 & 0.43979 \\
\hline & 5 & 0.56843 & 0.57416 & 0.54903 & 0.52123 & 0.51790 & 0.51668 \\
\hline \multirow{5}{*}{2} & 1 & 0.04860 & 0.04647 & 0.03807 & 0.03375 & 0.02991 & 0.02651 \\
\hline & 2 & 0.16002 & 0.15437 & 0.11249 & 0.08539 & 0.06749 & 0.05671 \\
\hline & 3 & 0.28897 & 0.28114 & 0.17834 & 0.15122 & 0.13437 & 0.12147 \\
\hline & 4 & 0.40934 & 0.39996 & 0.27766 & 0.24244 & 0.20903 & 0.18748 \\
\hline & 5 & 0.50955 & 0.49775 & 0.37315 & 0.31397 & 0.28271 & 0.26366 \\
\hline \multirow{5}{*}{3} & 1 & 0.03384 & 0.03337 & 0.03160 & 0.03079 & 0.02993 & 0.02928 \\
\hline & 2 & 0.09454 & 0.09263 & 0.08141 & 0.06986 & 0.05939 & 0.05415 \\
\hline & 3 & 0.18307 & 0.17988 & 0.12435 & 0.09689 & 0.08751 & 0.08245 \\
\hline & 4 & 0.27974 & 0.27567 & 0.17770 & 0.16298 & 0.14935 & 0.14074 \\
\hline & 5 & 0.37331 & 0.36820 & 0.25761 & 0.21300 & 0.18892 & 0.18024 \\
\hline
\end{tabular}

of ABAQUS. Figure 7 shows the radial displacements of the points $D_{2}, D_{3}$, and $D_{4}$ when the axial force $f_{u}\left(f_{u}=1 N\right)$ acts on the point $D_{1}$ of the ECE and PEC vessels.

The forced vibrations of the airtight vessels show almost the same resonance peak at the three test points of the airtight vessel. However, as shown in the Figure 7, when the axial force $F$ is acted, the displacement is the largest at point $D_{4}$, which is far from the point of action of the force, and point
$D_{2}$ is the next. This means that the influence of vibrations at the junction of the shell component is great when the airtight vessel is subjected to external action.

\section{Conclusions}

In this paper, the unified Jacobi-Ritz Method was presented and the free and forced vibrations of the airtight vessels consisting of elliptical, cylindrical, and paraboloidal shells 


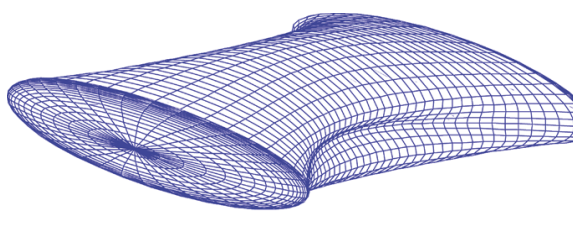

$\Omega_{1,2}=0.04754$

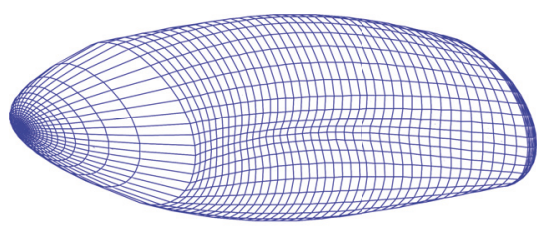

$\Omega_{1,2}=0.04230$

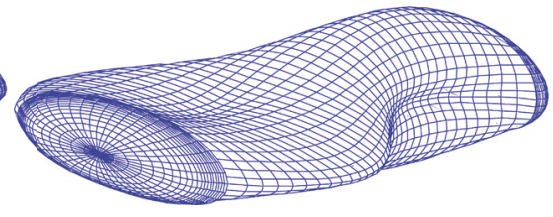

$\Omega_{2,2}=0.15815$

(a) ECE vessel

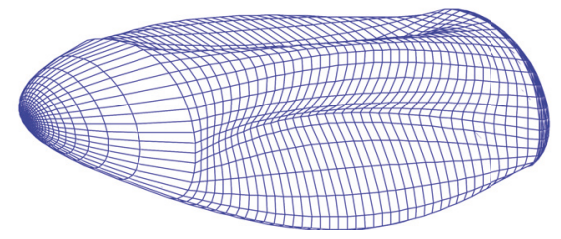

$\Omega_{1,3}=0.03247$

(b) PCE vessel

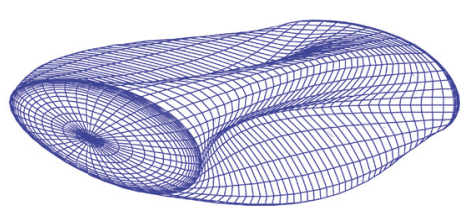

$\Omega_{1,3}=0.03332$

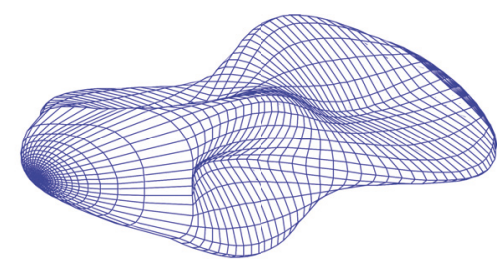

$\Omega_{2,3}=0.08817$

FIGURE 5: Some mode shapes of the ECE and PCE vessels.

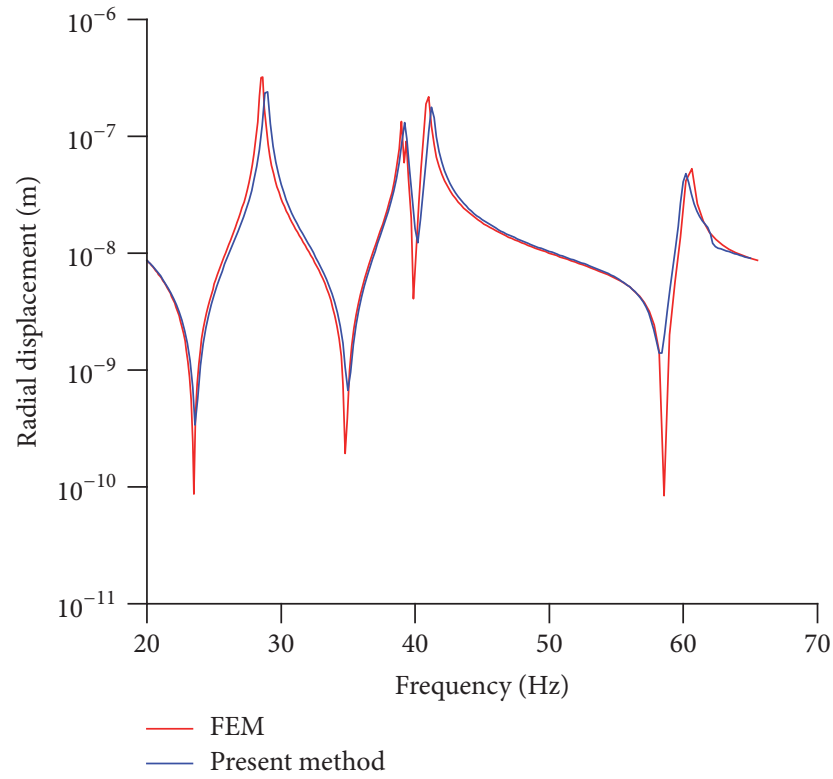

(a)

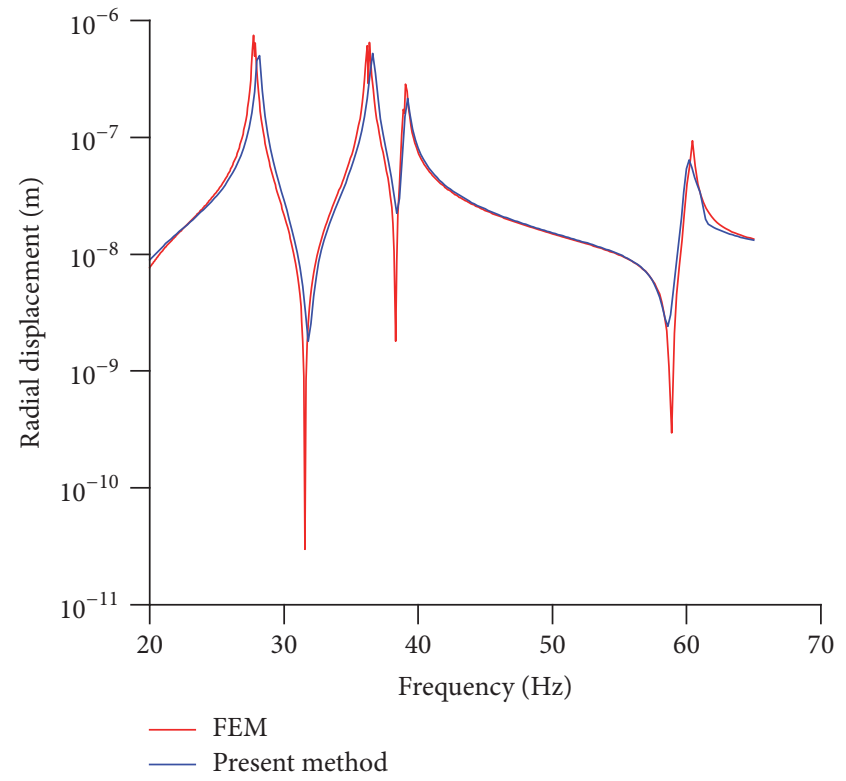

(b)

FIGURE 6: The radial displacements of point $D_{3}$ when the radial force $f_{w}$ acts on point $D_{2}$ of the ECE vessel and the PCE vessel: (a) ECE vessel, (b) PCE vessel.

were analyzed. In this study, Flügge's thin shell theory was adopted for the calculation model of vessels; the selection of displacement functions was generalized by the Jacobi polynomials. In addition, the continuous conditions at the interface were incorporated into the presented model by using the spring stiffness technique, and the accuracy of solution was improved by the domain decomposition method. The results of the proposed method show that this method can vouch faster convergence, high accuracy, and simplicity of calculation. Furthermore, the results of various free and 


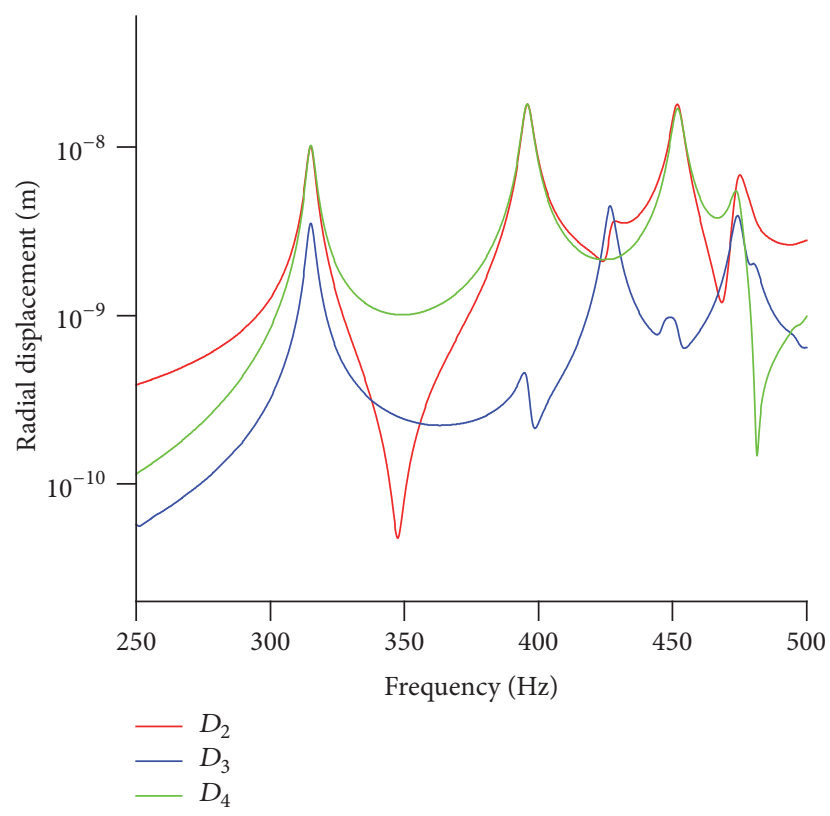

(a)

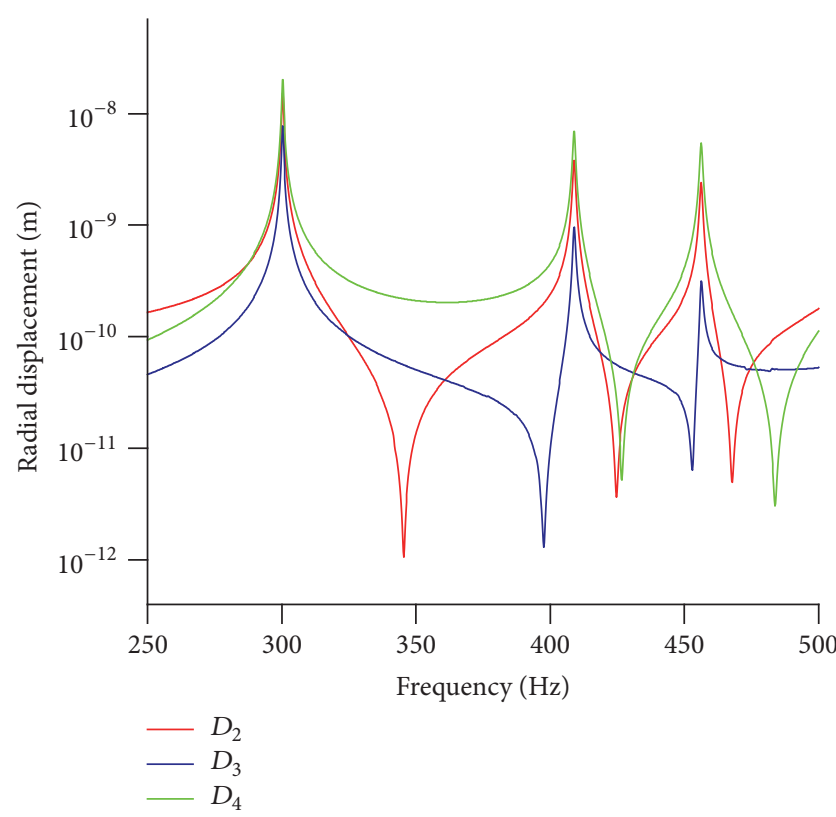

(b)

FIGURE 7: The radial displacements of the point $D_{2}, D_{3}$, and $D_{4}$ when the axial force $f_{u}$ acts on the point $D_{1}$ of the ECE and PEC vessels: (a) ECE vessel, (b) PCE vessel.

forced vibrations of the elliptical-cylindrical-elliptical and the paraboloidal-cylindrical-elliptical vessels were presented. We hope that these results can be used as benchmark data for future researchers.

\section{Appendix}

The generalized mass and stiffness matrix of the airtight cylindrical vessels used in (21) is given as

$$
\begin{aligned}
& M=\operatorname{diag}\left[M_{l}^{1}, M_{l}^{2}, \ldots, M_{l}^{N_{l}}, M_{c}^{1}, M_{c}^{2}, \ldots, M_{c}^{N_{c}}, M_{r}^{1}, M_{r}^{2},\right. \\
& \left.\ldots, M_{r}^{N_{r}}\right] \\
& M_{\xi}^{i}=\int_{\varphi_{\xi, i}}^{\varphi_{\xi, i+1}} \int_{0}^{2 \pi}\left[\begin{array}{ccc}
M_{\xi, u u} & 0 & 0 \\
0 & M_{\xi, v v} & 0 \\
0 & 0 & M_{\xi, w w}
\end{array}\right] A B d \varphi_{\xi} d \theta_{\xi} \\
& M_{\xi, u u}=\rho h U^{T} U, \\
& M_{\xi, v v}=\rho h V^{T} V, \\
& M_{\xi, w w}=\rho h W^{T} W, \\
& U=P_{m} \otimes C_{n}, \\
& V=P_{m} \otimes S_{n}, \\
& W=P_{m} \otimes C_{n}, \\
& P_{m}=\left[P_{0}^{(\alpha, \beta)}(\phi), P_{1}^{(\alpha, \beta)}(\phi), \ldots, P_{m}^{(\alpha, \beta)}(\phi), \ldots,\right. \\
& \left.\quad P_{M}^{(\alpha, \beta)}(\phi)\right]
\end{aligned}
$$

where the subscript $\xi(\xi=l, c, r)$ represents the left, cylindrical, and right shells and the superscript $i$ represents the $i$ th segment in the $\xi$ shell components.

$$
\begin{aligned}
& K=K_{\xi}+K_{c}+K_{s} \\
& K_{\xi}=\operatorname{diag}\left[K_{l}^{1}, K_{l}^{2}, \ldots, K_{l}^{N_{l}}, K_{c}^{1}, K_{c}^{2}, \ldots, K_{c}^{N_{c}}, K_{r}^{1}, K_{r}^{2}, \ldots,\right. \\
& \left.K_{r}^{N_{r}}\right] \\
& K_{\xi}^{i}=\int_{\varphi_{\xi, i}}^{\varphi_{\xi, i+1}} \int_{0}^{2 \pi}\left[\begin{array}{lll}
K_{\xi, u u} & K_{\xi, u v} & K_{\xi, u w} \\
K_{\xi, u v}^{T} & K_{\xi, v v} & K_{\xi, v w} \\
K_{\xi, u w}^{T} & K_{\xi, v w}^{T} & K_{\xi, w w}
\end{array}\right] A B d \varphi_{\xi} d \theta_{\xi} .
\end{aligned}
$$

Left and right shell:

$$
\begin{gathered}
K_{\xi, u u}=K_{E} u u_{\mathrm{Q} 0}+D_{G}\left(u u_{\mathrm{Q} 1}+u u_{\mathrm{Q} 2}\right) \\
K_{\xi, u v}=K_{E} u v_{\mathrm{Q} 0}+D_{G}\left(u v_{\mathrm{Q} 1}+u v_{\mathrm{Q} 2}\right) \\
K_{\xi, u w}=K_{E} u w_{\mathrm{Q} 0}+D_{G}\left(u w_{\mathrm{Q} 1}+u w_{\mathrm{Q} 2}\right)
\end{gathered}
$$


14

Shock and Vibration

$$
\begin{gathered}
K_{\xi, v v}=K_{E} v v_{\mathrm{Q} 0}+D_{G}\left(v v_{\mathrm{Q} 1}+v v_{\mathrm{Q} 2}\right) \\
K_{\xi, v w}=K_{E} v w_{\mathrm{Q} 0}+D_{G}\left(v w_{\mathrm{Q} 1}+v w_{\mathrm{Q} 2}\right) \\
K_{\xi, w w}=K_{E} w w_{\mathrm{Q} 0}+D_{G}\left(w w_{\mathrm{Q} 1}+w w_{\mathrm{Q} 2}\right),
\end{gathered}
$$

where $K_{E}=E h /\left(1-v^{2}\right), D_{G}=E h^{3} /\left[12\left(1-v^{2}\right)\right]$.

$$
\begin{aligned}
& u u_{\mathrm{Q} 0}=\frac{1}{R_{\varphi}^{2}} \frac{\partial U^{T}}{\partial \varphi} \frac{\partial U}{\partial \varphi}+\frac{1}{R_{\theta}^{2} T_{\varphi}^{2}} U^{T} U \\
& +\frac{\nu}{R_{\varphi} R_{\theta} T_{\varphi}}\left(\frac{\partial U^{T}}{\partial \varphi} U+U^{T} \frac{\partial U}{\partial \varphi}\right)+\frac{(1-\nu)}{2 R_{\theta}^{2} S_{\varphi}^{2}} \\
& \frac{\partial U^{T}}{\partial \theta} \frac{\partial U}{\partial \theta} \\
& u u_{\mathrm{Q} 1}=\frac{1}{R_{\varphi}^{2}}\left(U 1^{T} U 1+\frac{1}{R_{\theta}^{2} T_{\varphi}^{2}} U^{T} U\right. \\
& \left.+\frac{\nu}{R_{\theta} T_{\varphi}}\left(U 1^{T} U+U^{T} U 1\right)+\frac{(1-\nu)}{R_{\theta}^{2} S_{\varphi}^{2}} \frac{\partial U^{T}}{\partial \theta} \frac{\partial U}{\partial \theta}\right) \\
& u u_{\mathrm{Q} 2}=\left(\frac{1}{R_{\varphi}}-\frac{1}{R_{\theta}}\right)\left[\frac{1}{R_{\theta}^{2} T_{\varphi}^{2}}\left(\frac{2}{R_{\varphi}}-\frac{1}{R_{\theta}}\right) U^{T} U\right. \\
& \left.-\frac{1}{R_{\varphi}^{2}}\left(\frac{\partial U^{T}}{\partial \varphi} U 1+U 1^{T} \frac{\partial U}{\partial \varphi}\right)+\frac{1}{R_{\varphi}^{3}} \frac{\partial U^{T}}{\partial \varphi} \frac{\partial U}{\partial \varphi}\right] \\
& +\frac{(1-\nu)}{2 R_{\theta}^{2} S_{\varphi}^{2}}\left(\frac{-1}{R_{\varphi}^{2}}+\frac{-3}{R_{\varphi} R_{\theta}}+\frac{1}{R_{\theta}^{2}}\right) \frac{\partial U^{T}}{\partial \theta} \frac{\partial U}{\partial \theta} \\
& u v_{\mathrm{Q} 0}=\left(\frac{C_{\varphi}}{R_{\theta}^{2} S_{\varphi}^{2}} U+\frac{v}{R_{\varphi} R_{\theta} S_{\varphi}} \frac{\partial U}{\partial \varphi}\right)^{T} \frac{\partial V}{\partial \theta}+\frac{(1-\nu)}{2 R_{\theta} S_{\varphi}} \\
& \cdot \frac{\partial U}{\partial \theta}^{T} V 1 \\
& u v_{\mathrm{Q} 1}=\left(\frac{1}{R_{\varphi} R_{\theta} S_{\varphi}}\right)\left[\left(\frac{1}{R_{\theta}^{2} T_{\varphi}} U+\frac{\nu}{R_{\theta}} U 1\right)^{T} \frac{\partial V}{\partial \theta}\right. \\
& \left.+2(1-v){\frac{\partial U^{T}}{\partial \theta}}^{T} V 2\right] \\
& u v_{\mathrm{Q} 2}=\left(\frac{1}{R_{\varphi}}-\frac{1}{R_{\theta}}\right) \frac{C_{\varphi}}{R_{\varphi} R_{\theta}^{2} S_{\varphi}^{2}} U^{T} \frac{\partial V}{\partial \theta}-\frac{1-\nu}{2 R_{\theta} S_{\varphi}} \\
& \cdot \frac{\partial U^{T}}{\partial \theta}\left[\left(\frac{2}{R_{\varphi} R_{\theta}}+\frac{1}{R_{\theta}^{2}}\right) V 1+\left(\frac{1}{R_{\varphi}}+\frac{1}{R_{\theta}}\right) V 2\right] \\
& u w_{\mathrm{Q} 0}=\left[\left(\frac{1}{R_{\varphi}^{2}}+\frac{\nu}{R_{\varphi} R_{\theta}}\right) \frac{\partial U}{\partial \varphi}\right. \\
& \left.+\left(\frac{1}{R_{\theta}^{2} T_{\varphi}}+\frac{v}{R_{\varphi} R_{\theta} T_{\varphi}}\right) U\right]^{T} W
\end{aligned}
$$

$$
\begin{aligned}
& u w_{\mathrm{Q} 1}=-\frac{1}{R_{\varphi}} U 1^{T}\left(\frac{1}{R_{\varphi}} W 1+\frac{\nu}{R_{\theta} S_{\varphi}} W 2\right)-\frac{1}{R_{\varphi} R_{\theta} T_{\varphi}} \\
& \cdot U^{T}\left(\frac{1}{R_{\theta} S_{\varphi}} W 2+\frac{\nu}{R_{\varphi}} W 1\right)+\frac{4(1-\nu)}{R_{\varphi} R_{\theta}^{2} S_{\varphi}^{2}} \frac{\partial U^{T}}{\partial \theta} W 3 \\
& u w_{\mathrm{Q} 2}=\left(\frac{1}{R_{\varphi}}-\frac{1}{R_{\theta}}\right)\left\{\frac{1}{R_{\varphi}^{2}} \frac{\partial U^{T}}{\partial \varphi}\left(W 1+\frac{1}{R_{\varphi}} W\right)\right. \\
& -\frac{1}{R_{\varphi}^{2}} U 1^{T} W \\
& \left.+\frac{C_{\varphi}}{R_{\theta}^{2} S_{\varphi}^{2}} U^{T}\left[\left(\frac{1}{R_{\varphi}}-\frac{1}{R_{\theta}}\right) S_{\varphi} W-W 2\right]\right\}-\left(\frac{1}{R_{\varphi}}\right. \\
& \left.+\frac{1}{R_{\theta}}\right) \frac{(1-v)}{2 R_{\theta}^{2} S_{\varphi}^{2}} \frac{\partial U^{T}}{\partial \theta} W 3 \\
& v v_{\mathrm{Q} 0}=\frac{1}{R_{\theta}^{2} S_{\varphi}^{2}} \frac{\partial V^{T}}{\partial \theta} \frac{\partial V}{\partial \theta}+\frac{(1-\nu)}{2} V 1^{T} V 1 \\
& v v_{\mathrm{Q} 1}=\frac{1}{R_{\theta}^{4} S_{\varphi}^{2}} \frac{\partial V^{T}}{\partial \theta} \frac{\partial V}{\partial \theta}+\frac{(1-\nu)}{2} V 2^{T} V 2 \\
& v v_{\mathrm{Q} 2}=\left(\frac{1}{R_{\varphi} R_{\theta}^{3} S_{\varphi}^{2}}-\frac{1}{R_{\theta}^{4} S_{\varphi}^{2}}\right) \frac{\partial V^{T}}{\partial \theta} \frac{\partial V}{\partial \theta} \\
& -\frac{(1-v)}{2}\left[\left(\frac{1}{R_{\varphi}}+\frac{1}{R_{\theta}}\right)\left(V 1^{T} V 2+V 2^{T} V 1\right)\right. \\
& \left.+\left(\frac{1}{R_{\varphi}^{2}}-\frac{1}{R_{\varphi} R_{\theta}}+\frac{1}{R_{\theta}^{2}}\right) V 1^{T} V 1\right] \\
& v w_{\mathrm{Q} 0}=\left(\frac{v}{R_{\varphi} R_{\theta} S_{\varphi}}+\frac{1}{R_{\theta}^{2} S_{\varphi}}\right) \frac{\partial V^{T}}{\partial \theta} W \\
& v w_{\mathrm{Q} 1}=-\frac{1}{R_{\theta}^{2} S_{\varphi}} \frac{\partial V^{T}}{\partial \theta}\left(\frac{1}{R_{\theta} S_{\varphi}} W 2+\frac{\nu}{R_{\varphi}} W 1\right) \\
& +\frac{4(1-\nu)}{R_{\theta} S_{\varphi}} V 2^{T} W 3 \\
& v w_{\mathrm{Q} 2}=-\frac{1}{R_{\theta}^{2} S_{\varphi}^{2}}\left(\frac{1}{R_{\varphi}}-\frac{1}{R_{\theta}}\right) \frac{\partial V^{T}}{\partial \theta} W 2 \\
& -\frac{(1-v)}{2 R_{\theta} S_{\varphi}}\left(\frac{1}{R_{\varphi}}+\frac{1}{R_{\theta}}\right) V 1^{T} W 3 \\
& w w_{\mathrm{Q} 0}=\frac{1}{R_{\varphi}^{2}}+\frac{1}{R_{\theta}^{2}}+\frac{2 \nu}{R_{\varphi} R_{\theta}} W^{T} W \\
& w w_{\mathrm{Q} 1}=\frac{1}{R_{\varphi}^{2}} W 1^{T} W 1+\frac{1}{R_{\theta}^{2} S_{\varphi}^{2}} W 2^{T} W 2 \\
& +\frac{\nu}{R_{\varphi} R_{\theta} S_{\varphi}}\left(W 1^{T} W 2+W 2^{T} W 1\right)+\frac{2(1-\nu)}{R_{\theta}^{2} S_{\varphi}^{2}} \\
& \text { - } W 3^{T} W 3
\end{aligned}
$$




$$
\begin{gathered}
w w_{\mathrm{Q} 2}=\left(\frac{1}{R_{\varphi}}-\frac{1}{R_{\theta}}\right)\left[\frac{1}{R_{\varphi}^{2}}\left(W^{T} W 1+W 1^{T} W\right)\right. \\
-\frac{1}{R_{\theta}^{2} S_{\varphi}}\left(W^{T} W 2+W 2^{T} W\right) \\
\left.+\left(\frac{1}{R_{\varphi}^{3}}-\frac{1}{R_{\theta}^{3}}\right) W^{T} W\right]
\end{gathered}
$$

where $C_{\varphi}=\cos (\varphi), S_{\varphi}=\sin (\varphi), T_{\varphi}=\tan (\varphi)$ :

$$
\begin{aligned}
& P 1=\left(\frac{1}{R_{\varphi}}-\frac{1}{R_{\theta}}\right), \\
& P 2=\left(\frac{1}{R_{\varphi}}+\frac{1}{R_{\theta}}\right), \\
& P 3=\left(\frac{1}{R_{\varphi}^{2}}-\frac{1}{R_{\varphi} R_{\theta}}+\frac{1}{R_{\theta}^{2}}\right)
\end{aligned}
$$$$
K_{\xi, u u}=K_{E}\left(\frac{\partial U^{T}}{\partial x} \frac{\partial U}{\partial x}+\frac{(1-\nu)}{2 R_{c}^{2}} \frac{\partial U^{T}}{\partial \theta} \frac{\partial U}{\partial \theta}\right)+D_{G}\left(\frac{(1-\nu)}{2 R_{c}^{4}} \frac{\partial U^{T}}{\partial \theta} \frac{\partial U}{\partial \theta}\right)
$$$$
K_{\xi, u v}=K_{E}\left(\frac{\nu}{R_{c}} \frac{\partial U^{T}}{\partial x} \frac{\partial V}{\partial \theta}+\frac{(1-\nu)}{2 R_{c}} \frac{\partial U^{T}}{\partial \theta} \frac{\partial V}{\partial x}\right)
$$$$
K_{\xi, u w}=K_{E} \frac{\nu}{R_{c}} \frac{\partial U^{T}}{\partial x} W-D_{G}\left(\frac{1}{2 R_{c}} \frac{\partial U^{T}}{\partial x} \frac{\partial^{2} W}{\partial x^{2}}-\frac{(1-v)}{2 R_{c}^{3}} \frac{\partial U^{T}}{\partial \theta} \frac{\partial^{2} W}{\partial x \partial \theta}\right)
$$$$
K_{\xi, v v}=K_{E}\left(\frac{(1-\nu)}{2} \frac{\partial V^{T}}{\partial x} \frac{\partial V}{\partial x}+\frac{1}{R_{c}^{2}} \frac{\partial V^{T}}{\partial \theta} \frac{\partial V}{\partial \theta}\right)+D_{G} \frac{3(1-\nu)}{2 R_{c}^{2}} \frac{\partial V^{T}}{\partial x} \frac{\partial V}{\partial x}
$$$$
K_{\xi, v w}=K_{E} \frac{1}{R_{c}^{2}} \frac{\partial V^{T}}{\partial \theta} W-D_{G}\left(\frac{\nu}{R_{c}^{2}} \frac{\partial V^{T}}{\partial \theta} \frac{\partial^{2} W}{\partial x^{2}}+\frac{3(1-\nu)}{2 R_{c}^{2}} \frac{\partial V^{T}}{\partial x} \frac{\partial^{2} W}{\partial x \partial \theta}\right)
$$$$
K_{\xi, w w}=K_{E} \frac{1}{R_{c}^{2}} W^{T} W+D_{G}\left[\frac{\partial^{2} W}{\partial x^{2}} \frac{\partial^{2} W}{\partial x^{2}}+\frac{\nu}{R_{c}^{2}}\left(\frac{\partial^{2} W^{T}}{\partial x^{2}} \frac{\partial^{2} W}{\partial \theta^{2}}+\frac{\partial^{2} W^{T}}{\partial \theta^{2}} \frac{\partial^{2} W}{\partial x^{2}}\right)+\frac{1}{R_{c}^{4}} \frac{\partial^{2} W}{\partial \theta^{2}} \frac{\partial^{2} W}{\partial \theta^{2}}+\frac{2(1-\nu)}{R_{c}^{2}}\right.
$$$$
\left.\frac{\partial^{2} W}{\partial x \partial \theta} \frac{\partial^{2} W}{\partial x \partial \theta}+\frac{1}{R_{c}^{4}} W^{T} W+\frac{1}{R_{c}^{4}}\left(W^{T} \frac{\partial^{2} W}{\partial \theta^{2}}+\frac{\partial^{2} W^{T}}{\partial \theta^{2}} W\right)\right]
$$

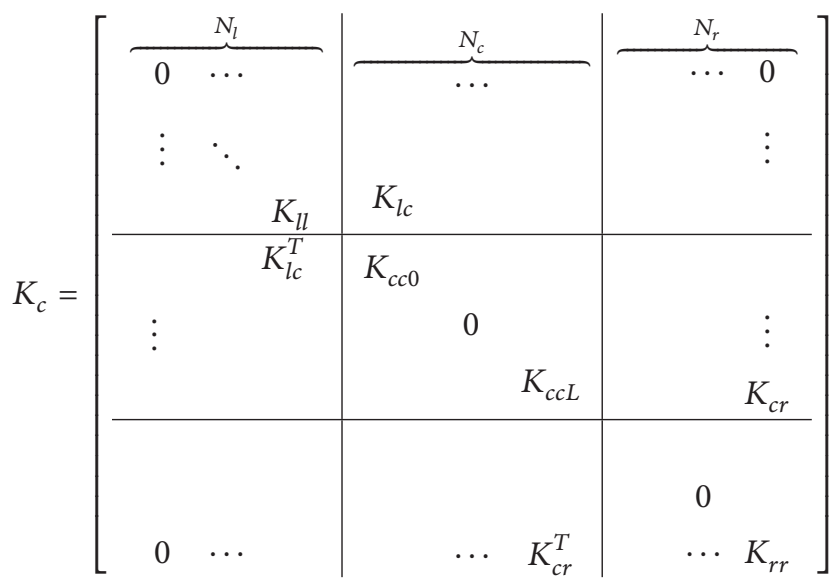

$$
\begin{aligned}
U 1 & =\frac{d R_{\varphi}}{d \varphi} U+\frac{1}{R_{\varphi}} \frac{\partial U}{\partial \varphi}, \\
V 1 & =\frac{1}{R_{\varphi}} \frac{\partial V}{\partial \varphi}-\frac{1}{R_{\theta} T_{\varphi}} V, \\
V 2 & =\frac{1}{R_{\theta}}\left(\frac{1}{R_{\varphi}} \frac{\partial V}{\partial \varphi}-\frac{1}{R_{\theta} T_{\varphi}} V\right)=\frac{1}{R_{\theta}} V 1
\end{aligned}
$$$$
W 1=\frac{d R_{\varphi}}{d \varphi} \frac{\partial W}{\partial \varphi}+\frac{1}{R_{\varphi}} \frac{\partial^{2} W}{\partial \varphi^{2}},
$$$$
W 2=\frac{1}{R_{\theta} S_{\varphi}} \frac{\partial^{2} W}{\partial \varphi^{2}}+\frac{C_{\varphi}}{R_{\varphi}} \frac{\partial W}{\partial \varphi},
$$$$
W 3=\frac{1}{R_{\theta} T_{\varphi}} \frac{\partial W}{\partial \theta}-\frac{1}{R_{\varphi}} \frac{\partial^{2} W}{\partial \varphi \partial \theta} .
$$

Cylindrical shells $\xi(\xi=c)$ : 


$$
\begin{aligned}
& K_{l l}=\int_{0}^{2 \pi}\left[\begin{array}{ccc}
K_{l l, u u} & 0 & K_{l l, u w} \\
0 & K_{l l, v v} & 0 \\
K_{l l, u w}^{T} & 0 & K_{l l, w w}
\end{array}\right]_{\varphi=\varphi_{l 1}} R_{c} d \theta, \\
& K_{l c}=\int_{0}^{2 \pi}\left[\begin{array}{ccc}
K_{l c, u u} & 0 & K_{l c, u w} \\
0 & K_{l c, v v} & 0 \\
K_{l c, w u} & 0 & K_{l c, w w}
\end{array}\right]_{\varphi=\varphi_{11}, x=0} R_{c} d \theta \\
& K_{c c 0, L}=\int_{0}^{2 \pi}\left[\begin{array}{ccc}
K_{c c, u u} & 0 & 0 \\
0 & K_{c c, v v} & 0 \\
0 & 0 & K_{c c, w w}
\end{array}\right]_{x=0, x=L_{c}} R_{c} d \theta, \\
& K_{r r}=\int_{0}^{2 \pi}\left[\begin{array}{ccc}
K_{r r, u u} & 0 & K_{r r, u w} \\
0 & K_{r r, v v} & 0 \\
K_{r r, u w}^{T} & 0 & K_{r r, w w}
\end{array}\right]_{\varphi=\varphi_{r 1}} R_{c} d \theta, \\
& K_{c r}=\int_{0}^{2 \pi}\left[\begin{array}{ccc}
K_{c r, u u} & 0 & K_{c r, u w} \\
0 & K_{c r, v v} & 0 \\
K_{c r, w u} & 0 & K_{c r, w w}
\end{array}\right]_{x=L_{c}, \varphi=\varphi_{r 1}} R_{c} d \theta \\
& K_{l l, u u}=\left(k_{u} \sin ^{2} \varphi_{l 1}+k_{w} \cos ^{2} \varphi_{l 1}+k_{r} \frac{1}{R_{\varphi}^{2}}\right) U_{\varphi}^{T} U_{\varphi} \\
& K_{l l, v v}=k_{v} V_{\varphi}^{T} V_{\varphi} \\
& K_{l l, w w}=\left(k_{u} \cos ^{2} \varphi_{l 1}+k_{w} \sin ^{2} \varphi_{l 1}\right) W_{\varphi}^{T} W_{\varphi}+k_{r} \frac{1}{R_{\varphi}^{2}} \frac{\partial W_{\varphi}{ }^{T}}{\partial \varphi} \frac{\partial W_{\varphi}}{\partial \varphi} \\
& K_{l l, u w}=\left(-k_{u}+k_{w}\right) \cos \varphi_{l 1} \sin \varphi_{l 1} U_{\varphi}{ }^{T} W_{\varphi}-k_{r} \frac{1}{R_{\varphi}^{2}} U_{\varphi}{ }^{T} \frac{\partial W_{\varphi}}{\partial \varphi} \\
& K_{l c, u u}=-k_{u} \sin \varphi_{l 1} U_{\varphi}{ }^{T} U_{x} \\
& K_{l c, v v}=-k_{v} V_{\varphi}^{T} V_{x} \\
& K_{l c, w w}=-k_{w} \sin \varphi_{l 1} W_{\varphi}^{T} W_{x}-k_{r} \frac{1}{R_{\varphi}} \frac{\partial W_{\varphi}^{T}}{\partial \varphi} \frac{\partial W_{x}}{\partial x} \\
& K_{l c, u w}=-k_{w} \cos \varphi_{l 1} U_{\varphi}{ }^{T} W_{x}+k_{r} \frac{1}{R_{\varphi}} U_{\varphi}{ }^{T} \frac{\partial W_{x}}{\partial x} \\
& K_{l c, w u}=k_{w} \cos \varphi_{l 1} W_{\varphi}^{T} U_{x} \\
& K_{c c, u u}=k_{u} U_{x}^{T} U_{x} \\
& K_{c c, v v}=k_{v} V_{x}^{T} V_{x} \\
& K_{c c, w w}=k_{w} W_{x}^{T} W_{x}+k_{w} \frac{\partial W_{x}{ }^{T}}{\partial x} \frac{\partial W_{x}}{\partial x}
\end{aligned}
$$




$$
\begin{aligned}
& K_{c r}=\int_{0}^{2 \pi}\left[\begin{array}{cccccc}
K_{c c, u u} & 0 & K_{c c, u w} & K_{c r, u u} & & K_{c r, u w} \\
0 & K_{c c, v v} & 0 & & K_{c r, v v} & \\
K_{c c, u w}^{T} & 0 & K_{c c, w w} & K_{c r, w u} & & K_{c r, w w} \\
K_{c r, u u}^{T} & & K_{c r, w u}^{T} & K_{r r, u u} & & \\
& K_{c r, v v}^{T} & & & K_{r r, v v} & \\
K_{c r, u w}^{T} & & K_{c r, w w}^{T} & & & K_{r r, w w}
\end{array}\right]_{x=L_{c}, \varphi=\varphi_{r 1}} \\
& K_{c c, u u}=k_{u} U_{x}^{T} U_{x} \\
& K_{c c, v v}=k_{v} V_{x}^{T} V_{x} \\
& K_{c c, w w}=k_{w} W_{x}^{T} W_{x}+k_{w} \frac{\partial W_{x}^{T}}{\partial x} \frac{\partial W_{x}}{\partial x} \\
& K_{c r, u u}=k_{u} \sin \varphi_{r 1} U_{x}{ }^{T} U_{\varphi} \\
& K_{c r, v v}=-k_{v} V_{x}^{T} V_{\varphi} \\
& K_{c r, w w}=-k_{w} \sin \varphi_{r 1} W_{x}^{T} W_{\varphi}-k_{r} \frac{1}{R_{\varphi}} \frac{\partial W_{x}{ }^{T}}{\partial x} \frac{\partial W_{\varphi}}{\partial \varphi} \\
& K_{c r, w u}=-k_{w} \cos \varphi_{r 1} W_{x}^{T} U_{\varphi}+k_{r} \frac{1}{R_{\varphi}} \frac{\partial W_{x}}{\partial x} U_{\varphi}^{T} \\
& K_{c r, u w}=k_{w} \cos \varphi_{r 1} U_{x}{ }^{T} W_{\varphi} \\
& K_{r r, u u}=\left(k_{u} \sin ^{2} \varphi_{r 1}+k_{w} \cos ^{2} \varphi_{r 1}+k_{r} \frac{1}{R_{\varphi}^{2}}\right) U_{\varphi}^{T} U_{\varphi} \\
& K_{r r, v v}=k_{v} V_{\varphi}^{T} V_{\varphi} \\
& K_{r r, w w}=\left(k_{u} \cos ^{2} \varphi_{r 1}+k_{w} \sin ^{2} \varphi_{r 1}\right) W_{\varphi}^{T} W_{\varphi}+k_{r} \frac{1}{R_{\varphi}^{2}} \frac{\partial W_{\varphi}{ }^{T}}{\partial \varphi} \frac{\partial W_{\varphi}}{\partial \varphi} \\
& K_{r r, u w}=-\left(k_{u}-k_{w}\right) \cos \varphi_{r 1} \sin \varphi_{r 1} U_{\varphi}{ }^{T} W_{\varphi}-k_{r} \frac{1}{R_{\varphi}^{2}} U_{\varphi}{ }^{T} \frac{\partial W_{\varphi}}{\partial \varphi} \\
& K_{s}=\operatorname{diag}\left[K_{s}^{1}, K_{s}^{2}, \ldots, K_{s}^{N_{l}}, K_{s}^{1}, K_{s}^{2}, \ldots, K_{s}^{N_{c}}, K_{s}^{1}, K_{s}^{2}, \ldots, K_{s}^{N_{r}}\right] \\
& K_{s}^{i}=\int_{0}^{2 \pi}\left[\begin{array}{ccc|ccc}
K_{u_{i} u_{i}} & 0 & K_{u_{i} w_{i}} & K_{u_{i} u_{i+1}} & & K_{u_{i} w_{i+1}} \\
0 & K_{v_{i} v_{i}} & 0 & & K_{v_{i} v_{i+1}} & \\
K_{u_{i} w_{i}}^{T} & 0 & K_{w_{i} w_{i}} & K_{w_{i} u_{i+1}} & & K_{w_{i} w_{i+1}} \\
\hline K_{u_{i} u_{i+1}}^{T} & & K_{w_{i} u_{i+1}}^{T} & K_{u_{i+1} u_{i+1}} & & K_{u_{i+1} w_{i+1}} \\
& K_{v_{i} v_{i+1}}^{T} & & & K_{v_{i+1} v_{i+1}} & \\
K_{u_{i} w_{i+1}}^{T} & & K_{w_{i} w_{i+1}}^{T} & K_{u_{i+1} w_{i+1}}^{T} & & K_{w_{i+1} w_{i+1}}
\end{array}\right] B d \theta
\end{aligned}
$$


Left and right shell:

$$
\begin{aligned}
& K_{u_{i} u_{i}}=\left(k_{u}+k_{r} \frac{1}{R_{\varphi}^{2}}\right) U_{i}^{T} U_{i} \\
& K_{u_{i} w_{i}}=-k_{r} \frac{1}{R_{\varphi}^{2}} U_{i}^{T} \frac{\partial W_{i}}{\partial \varphi} \\
& K_{v_{i} v_{i}}=k_{v} V_{i}^{T} V_{i} \\
& K_{w_{i} w_{i}}=k_{w} W_{i}^{T} W_{i}+k_{r} \frac{1}{R_{\varphi}^{2}} \frac{\partial W_{i}^{T}}{\partial \varphi} \frac{\partial W_{i}}{\partial \varphi} \\
& K_{u_{i} u_{i+1}}=-\left(k_{u}+k_{r} \frac{1}{R_{\varphi}^{2}}\right) U_{i}^{T} U_{i+1} \\
& K_{u_{i} w_{i+1}}=k_{r} \frac{1}{R_{\varphi}^{2}} U_{i}^{T} \frac{\partial W_{i+1}}{\partial \varphi} \\
& K_{w_{i} u_{i+1}}=k_{r} \frac{1}{R_{\varphi}^{2}} \frac{\partial W_{i}^{T}}{\partial \varphi} U_{i+1} \\
& K_{v_{i} v_{i+1}}=-k_{v} V_{i}^{T} V_{i+1} \\
& K_{w_{i} w_{i+1}}=-k_{w} W_{i}^{T} W_{i-1}-k_{r} \frac{1}{R_{\varphi}^{2}} \frac{\partial W_{i}^{T}}{\partial \varphi} \frac{\partial W_{i+1}}{\partial \varphi} \\
& K_{u_{i+1} u_{i+1}}=\left(k_{u}+k_{r} \frac{1}{R_{\varphi}^{2}}\right) U_{i+1}^{T} U_{i+1} \\
& K_{u_{i+1} w_{i+1}}=-k_{r} \frac{1}{R_{\varphi}^{2}} U_{i+1}^{T} \frac{\partial W_{i+1}}{\partial \varphi} \\
& K_{v_{i+1} v_{i+1}}=k_{v} V_{i+1}^{T} V_{i+1} \\
& K_{w_{i+1} w_{i+1}}=k_{w} W_{i+1}^{T} W_{i+1}+k_{r} \frac{1}{R_{\varphi}^{2}} \frac{\partial W_{i+1}^{T}}{\partial \varphi} \frac{\partial W_{i+1}}{\partial \varphi} .
\end{aligned}
$$

Cylindrical shell:

$$
\begin{aligned}
K_{u_{i} u_{i}} & =k_{u} U_{i}^{T} U_{i} \\
K_{u_{i} w_{i}} & =0 \\
K_{v_{i} v_{i}} & =k_{v} V_{i}^{T} V_{i} \\
K_{w_{i} w_{i}} & =k_{w} W_{i}^{T} W_{i}+k_{r} \frac{\partial W_{i}^{T}}{\partial \varphi} \frac{\partial W_{i}}{\partial \varphi} \\
K_{u_{i} u_{i+1}} & =-k_{u} U_{i}^{T} U_{i+1} \\
K_{u_{i} w_{i+1}} & =0 \\
K_{w_{i} u_{i+1}} & =0
\end{aligned}
$$

$$
\begin{aligned}
K_{v_{i} v_{i+1}} & =-k_{v} V_{i}^{T} V_{i+1} \\
K_{w_{i} w_{i+1}} & =-k_{w} W_{i}^{T} W_{i-1}-k_{r} \frac{\partial W_{i}^{T}}{\partial \varphi} \frac{\partial W_{i+1}}{\partial \varphi} \\
K_{u_{i+1} u_{i+1}} & =k_{u} U_{i+1}^{T} U_{i+1} \\
K_{u_{i+1} w_{i+1}} & =0 \\
K_{v_{i+1} v_{i+1}} & =k_{v} V_{i+1}^{T} V_{i+1} \\
K_{w_{i+1} w_{i+1}} & =k_{w} W_{i+1}^{T} W_{i+1}+k_{r} \frac{\partial W_{i+1}^{T}}{\partial \varphi} \frac{\partial W_{i+1}}{\partial \varphi}
\end{aligned}
$$

where $C_{\varphi}=\cos (\varphi), S_{\varphi}=\sin (\varphi), T_{\varphi}=\tan (\varphi)$.

$$
\begin{aligned}
& \mathbf{X}=\left[U_{m n, \xi}^{i}, V_{m n, \xi}^{i}, W_{m n, \xi}^{i}\right]^{T} \\
& \mathbf{F}=\left[f_{u, m n, \xi}^{i}, f_{v, m n, \xi}^{i}, f_{w, m n, \xi}^{i}\right]^{T} .
\end{aligned}
$$

\section{Conflicts of Interest}

The authors declare that they have no conflicts of interest.

\section{Acknowledgments}

This study was funded by National Natural Science Foundation of China (no. 51209052), National key Research and Development program (2016YFC0303406), Fundamental Research Funds for the Central University (HEUCFD1515, HEUCFM170113), High Technology Ship Funds of Ministry of Industry and Information of China, Assembly forecasting fund (no. 6140210020105), China Postdoctoral Science Foundation (no. 2014M552661), and Major Innovation Projects Of High Technology Ship Funds of Ministry of Industry and Information of China.

\section{References}

[1] A. W. Leissa, Vibration of the Shells NASA SP-288, US Government Printing Office, Wash, USA, 1973.

[2] M. S. Qatu, "Recent research advances in the dynamic behavior of shells: 1989-2000, Part 2: homogeneous shells," Applied Mechanics Reviews, vol. 55, no. 5, pp. 415-434, 2002.

[3] K. M. Liew and C. W. Lim, "Vibration of doubly-curved shallow shells," Acta Mechanica, vol. 114, pp. 95-119, 1996.

[4] D.-Y. Tan, "Free vibration analysis of shells of revolution," Journal of Sound and Vibration, vol. 213, no. 1, pp. 15-33, 1998.

[5] D. Tang, X. Yao, G. Wu, and Y. Peng, "Free and forced vibration analysis of multi-stepped circular cylindrical shells with arbitrary boundary conditions by the method of reverberation-ray matrix," Thin-Walled Structures, vol. 116, pp. 154-168, 2017.

[6] K. Xie, M. Chen, and Z. Li, "Free and Forced Vibration Analysis of Ring-Stiffened Conical-Cylindrical-Spherical Shells Through a Semi-Analytic Method," Journal of Vibration and Acoustics, vol. 139, no. 3, Article ID 031001, 2017. 
[7] K. Xie, M. Chen, and Z. Li, "An analytic method for free and forced vibration analysis of stepped conical shells with arbitrary boundary conditions," Thin-Walled Structures, vol. 111, pp. 126137, 2017.

[8] M. S. Qatu and E. Asadi, "Vibration of doubly curved shallow shells with arbitrary boundaries," Applied Acoustics, vol. 73, no. 1, pp. 21-27, 2012.

[9] G. J. Zhang, T. Y. Li, X. Zhu, J. Yang, and Y. Y. Miao, "Free and forced vibration characteristics of submerged finite elliptic cylindrical shell," Ocean Engineering, vol. 129, pp. 92-106, 2017.

[10] S. Jiang, T. Yang, W. L. Li, and J. Du, "Vibration analysis of doubly curved shallow shells with elastic edge restraints," Journal of Vibration and Acoustics, vol. 135, article 034502, no. 3, 2013.

[11] A. Messina, "Free vibrations of multilayered doubly curved shells based on a mixed variational approach and global piecewise-smooth functions," International Journal of Solids and Structures, vol. 40, no. 12, pp. 3069-3088, 2003.

[12] R. Poultangari and M. Nikkhah-Bahrami, "A Vectorial-Wave Method for free and forced vibration analysis of extra thin cylindrical shells with boundary discrete damping," Journal of Mechanical Science and Technology, vol. 30, no. 7, pp. 3019-3030, 2016.

[13] M. Chen, K. Xie, W. Jia, and K. Xu, "Free and forced vibration of ring-stiffened conical-cylindrical shells with arbitrary boundary conditions," Ocean Engineering, vol. 108, pp. 241-256, 2015.

[14] F. Tornabene, "2-D GDQ solution for free vibrations of anisotropic doubly-curved shells and panels of revolution," Composite Structures, vol. 93, no. 7, pp. 1854-1876, 2011.

[15] F. Tornabene, N. Fantuzzi, and M. Bacciocchi, "Free vibrations of free-form doubly-curved shells made of functionally graded materials using higher-order equivalent single layer theories," Composites Part B: Engineering, vol. 67, pp. 490-509, 2014.

[16] F. Tornabene, N. Fantuzzi, E. Viola, and A. J. M. Ferreira, "Radial basis function method applied to doubly-curved laminated composite shells and panels with a General Higher-order Equivalent Single Layer formulation," Composites Part B: Engineering, vol. 55, pp. 642-659, 2013.

[17] M. Chen, K. Xie, K. Xu, and P. Yu, "Wave based method for free and forced vibration analysis of cylindrical shells with discontinuity in thickness," Journal of Vibration and Acoustics, vol. 137, article 051004, no. 5, 2015.

[18] X. Ma, G. Jin, Y. Xiong, and Z. Liu, "Free and forced vibration analysis of coupled conical-cylindrical shells with arbitrary boundary conditions," International Journal of Mechanical Sciences, vol. 88, pp. 122-137, 2014.

[19] L. Dozio, "Natural frequencies of sandwich plates with FGM core via variable-kinematic 2-D Ritz models," Composite Structures, vol. 96, pp. 561-568, 2013.

[20] T. Ye, G. Jin, and Y. Zhang, "Vibrations of composite laminated doubly-curved shells of revolution with elastic restraints including shear deformation, rotary inertia and initial curvature," Composite Structures, vol. 133, pp. 202-225, 2015.

[21] G. Jin, T. Ye, X. Wang, and X. Miao, "A unified solution for the vibration analysis of FGM doubly-curved shells of revolution with arbitrary boundary conditions," Composites Part B: Engineering, vol. 89, pp. 230-252, 2016.

[22] L. Dozio, "A hierarchical formulation of the state-space Levy's method for vibration analysis of thin and thick multilayered shells," Composites Part B: Engineering, vol. 98, pp. 97-107, 2016.
[23] L. Dozio and E. Carrera, "Ritz analysis of vibrating rectangular and skew multilayered plates based on advanced variablekinematic models," Composite Structures, vol. 94, no. 6, pp. 21182128, 2012.

[24] G. D. Galletly and J. Mistry, "The free vibrations of cylindrical shells with various end closures," Nuclear Engineering and Design, vol. 30, no. 2, pp. 249-268, 1974.

[25] T. Irie, G. Yamada, and Y. Muramoto, "Free vibration of joined conical-cylindrical shells," Journal of Sound and Vibration, vol. 95, no. 1, pp. 31-39, 1984.

[26] Y. Qu, Y. Chen, X. Long, H. Hua, and G. Meng, "Free and forced vibration analysis of uniform and stepped circular cylindrical shells using a domain decomposition method," Applied Acoustics, vol. 74, no. 3, pp. 425-439, 2013.

[27] S. K. Wong and K. Y. Sze, "Application of matched asymptotic expansions to the free vibration of a hermetic shell," Journal of Sound and Vibration, vol. 209, no. 4, pp. 593-607, 1998.

[28] M. Rougui, F. Moussaoui, and R. Benamar, "Geometrically nonlinear free and forced vibrations of simply supported circular cylindrical shells: A semi-analytical approach," International Journal of Non-Linear Mechanics, vol. 42, no. 9, pp. 1102-1115, 2007.

[29] E. Efraim and M. Eisenberger, "Exact vibration frequencies of segmented axisymmetric shells," Thin-Walled Structures, vol. 44, no. 3, pp. 281-289, 2006.

[30] M. Caresta and N. J. Kessissoglou, "Free vibrational characteristics of isotropic coupled cylindrical-conical shells," Journal of Sound and Vibration, vol. 329, no. 6, pp. 733-751, 2010.

[31] J. Lee, "Free vibration analysis of a hermetic capsule by pseudospectral method," Journal of Mechanical Science and Technology, vol. 26, no. 4, pp. 1011-1015, 2012.

[32] J. Lee, "Free vibration analysis of joined spherical-cylindrical shells by matched Fourier-Chebyshev expansions," International Journal of Mechanical Sciences, vol. 122, pp. 53-62, 2017.

[33] Y. Qu, Y. Chen, X. Long, H. Hua, and G. Meng, "A modified variational approach for vibration analysis of ring-stiffened conical-cylindrical shell combinations," European Journal of Mechanics - A/Solids, vol. 37, pp. 200-215, 2013.

[34] Y. Qu, S. Wu, Y. Chen, and H. Hua, "Vibration analysis of ringstiffened conical-cylindrical-spherical shells based on a modified variational approach," International Journal of Mechanical Sciences, vol. 69, pp. 72-84, 2013.

[35] S. Wu, Y. Qu, and H. Hua, "Vibration characteristics of a spherical-cylindrical-spherical shell by a domain decomposition method," Mechanics Research Communications, vol. 49, pp. 17-26, 2013.

[36] S. Wu, Y. Qu, and H. Hua, "Vibrations characteristics of joined cylindrical-spherical shell with elastic-support boundary conditions," Journal of Mechanical Science and Technology, vol. 27, no. 5, pp. 1265-1272, 2013.

[37] D. Shi, X. Lv, Q. Wang, and Q. Liang, "A unified solution for free vibration of orthotropic annular sector thin plates with general boundary conditions, internal radial line and circumferential arc supports," Journal of Vibroengineering, vol. 18, no. 1, pp. 361377, 2016.

[38] T. Warburton and M. Embree, "The role of the penalty in the local discontinuous Galerkin method for Maxwell's eigenvalue problem," Computer Methods Applied Mechanics and Engineering, vol. 195, no. 25-28, pp. 3205-3223, 2006. 
[39] D. Sármány, F. Izsák, and J. J. van der Vegt, “Optimal penalty parameters for symmetric discontinuous Galerkin discretisations of the time-harmonic Maxwell equations," Journal of Scientific Computing, vol. 44, no. 3, pp. 219-254, 2010.

[40] L. E. Monterrubio, "Free vibration of shallow shells using the Rayleigh-Ritz method and penalty parameters," Proceedings of the Institution of Mechanical Engineers, Part C: Journal of Mechanical Engineering Science, vol. 223, no. 10, pp. 2263-2272, 2009.

[41] D. Shi, Q. Liang, Q. Wang, and X. Teng, "A unified solution for free vibration of orthotropic circular, annular and sector plates with general boundary conditions," Journal of Vibroengineering, vol. 18, no. 5, pp. 3138-3152, 2016.

[42] Q. Wang, B. Qin, D. Shi, and Q. Liang, "A semi-analytical method for vibration analysis of functionally graded carbon nanotube reinforced composite doubly-curved panels and shells of revolution," Composite Structures, vol. 174, pp. 87-109, 2017.

[43] Q. Wang, D. Shi, and Q. Liang, "Free vibration analysis of axially loaded laminated composite beams with general boundary conditions by using a modified Fourier-Ritz approach," Journal of Composite Materials, vol. 50, no. 15, pp. 2111-2135, 2016.

[44] Q. Wang, D. Shi, Q. Liang, and F. e Ahad, "A unified solution for free in-plane vibration of orthotropic circular, annular and sector plates with general boundary conditions," Applied Mathematical Modelling: Simulation and Computation for Engineering and Environmental Systems, vol. 40, no. 21-22, pp. 9228-9253, 2016.

[45] H. Zhang, D. Shi, and Q. Wang, "An improved Fourier series solution for free vibration analysis of the moderately thick laminated composite rectangular plate with non-uniform boundary conditions," International Journal of Mechanical Sciences, vol. 121, pp. 1-20, 2017.

[46] H. Zhang, D. Shi, Q. Wang, and B. Qin, "Free vibration of functionally graded parabolic and circular panels with general boundary conditions," Curved and Layered Structures, vol. 4, no. 1, pp. 52-84, 2017.

[47] K. Zhang, D. Shi, W. Wang, and Q. Wang, "Mechanical characterization of hybrid lattice-to-steel joint with pyramidal CFRP truss for marine application," Composite Structures, vol. 160, pp. 1198-1204, 2017.

[48] A. H. Bhrawy, T. M. Taha, and J. A. Machado, "A review of operational matrices and spectral techniques for fractional calculus," Nonlinear Dynamics, vol. 81, no. 3, pp. 1023-1052, 2015. 


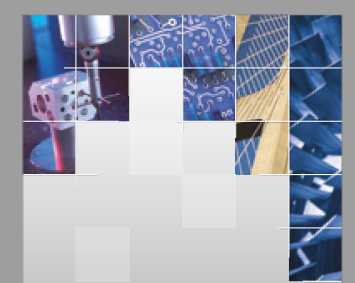

\section{Enfincering}
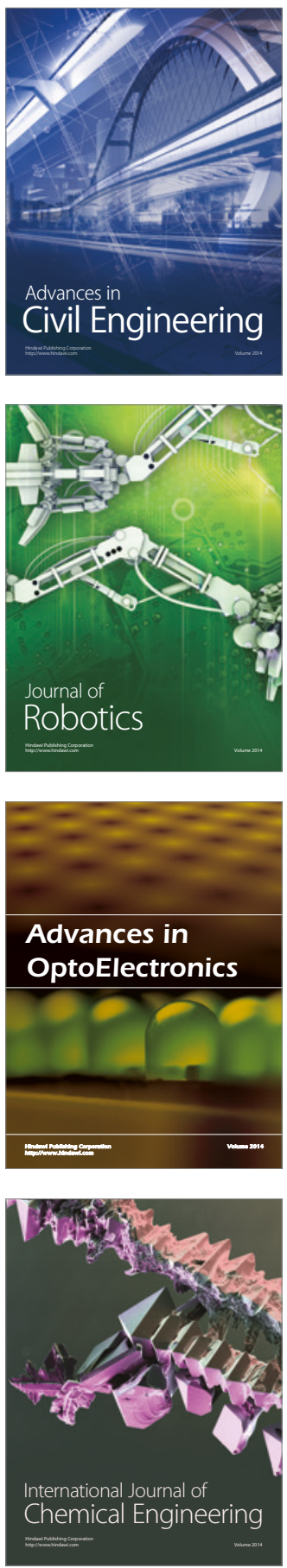

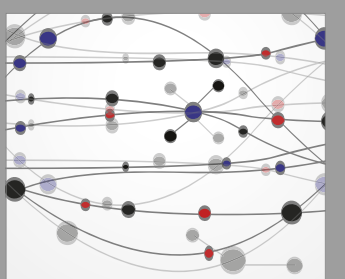

The Scientific World Journal

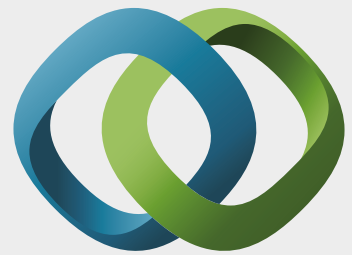

\section{Hindawi}

Submit your manuscripts at

https://www.hindawi.com
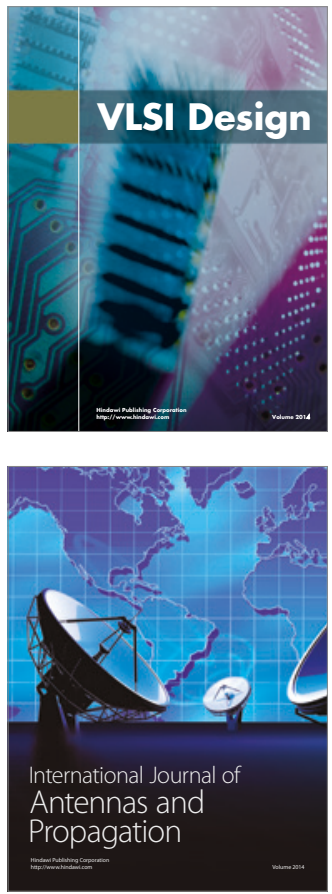

\section{Rotating}

Machinery
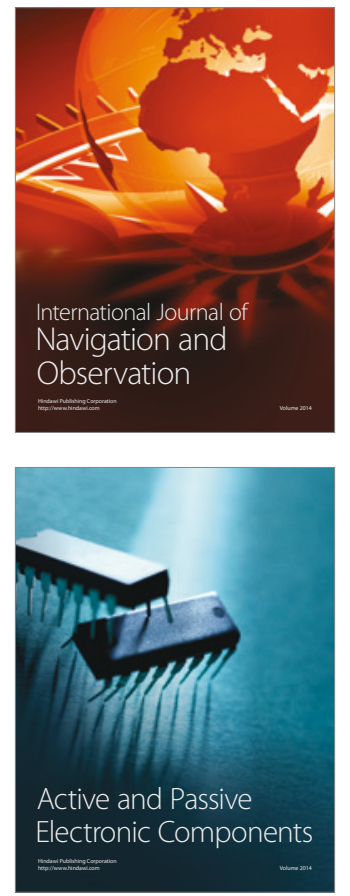

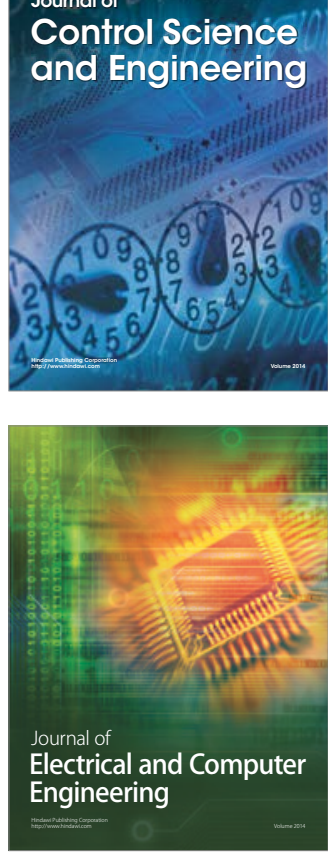

Distributed

Journal of

Control Science

and Engineering
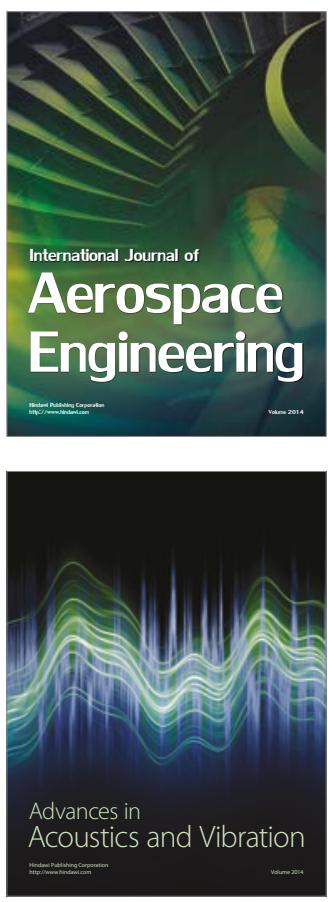

Sensor Networks 